\title{
Die drei Arenen der neuen Ungleichheitskonflikte. Eine sozialstrukturelle Positionsbestimmung der Einstellungen zu Umverteilung, Migration und sexueller Diversität
}

\author{
Steffen Mau • Thomas Lux • Fabian Gülzau
}

Online publiziert: 1. Dezember 2020

(C) Der/die Autor(en) 2020

Zusammenfassung In vielen westlichen Gesellschaften wird die Verteilungsfrage wieder stärker diskutiert. Mit den Debatten um Identitätspolitik und Migration sind aber weitere Konflikte in den Vordergrund getreten, sodass nicht mehr nur materielle Ungleichheitsfragen im Fokus stehen. In den wissenschaftlichen und publizistischen Debatten ist häufig von einer neuen Lagerbildung die Rede, bei der sich die bessergestellten Gruppen für Migration und die Anerkennung von Diversität aussprächen, während die schlechtergestellten Schichten dem ablehnend gegenüberstünden. Damit läge diese Konfliktkonstellation anders als der klassische Verteilungskonflikt, bei dem die oberen Statuslagen eine größere Ungleichheitstoleranz aufweisen als die unteren, eher ungleichheitskritischen Lagen. Im vorliegenden Aufsatz wird ein konzeptioneller Rahmen entwickelt, der über diese Zwei-Lager-Theorie hinausgeht und systematisch zwischen drei Dimensionen der Ungleichheitskonflikte und -einstellungen unterscheidet: (1) die klassischen Oben-Unten-Ungleichheiten, die sich auf die ökonomische Ressourcenverteilung beziehen, (2) die Wir-Sie-Ungleichheiten, die die gesellschaftliche Anerkennung von Diversität umfassen, und (3) die Innen-Außen-Ungleichheiten, die um den Grad an Offenheit gegenüber Zuwanderergruppen kreisen. Auf der Basis der SOEP-Innovations-Stichprobe aus dem Jahr 2017 lässt sich zeigen, dass es sich bei diesen Einstellungen um relativ eigenstän-

Zusatzmaterial online Zusätzliche Informationen sind in der Online-Version dieses Artikels (https:// doi.org/10.1007/s11609-020-00420-8) enthalten.

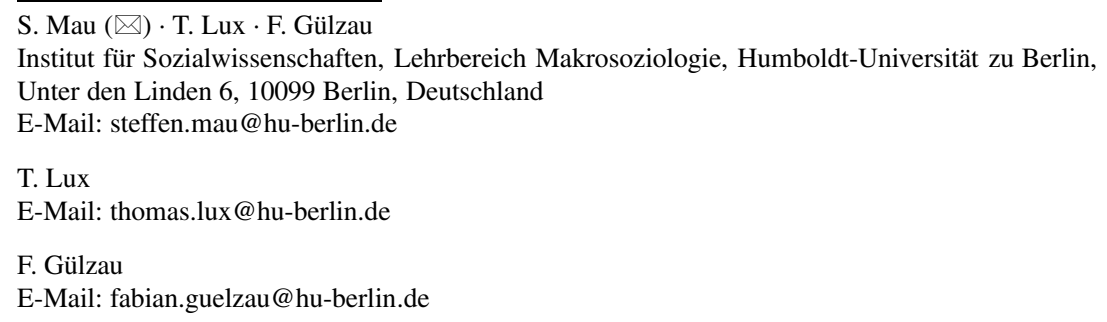


dige Dimensionen handelt. Beim Thema der ökonomischen Ressourcenverteilung sind dabei die unteren Statuslagen ungleichheitskritischer positioniert als die oberen Lagen; bei den beiden neueren Konfliktthemen ist das Bild jedoch genau umgekehrt. Allerdings sind nur beim Migrationsthema die Einstellungen wirklich polarisiert, ansonsten gibt es wenige Hinweise auf eine klare Lagerbildung, wie sie in der Literatur und der öffentlichen Diskussion oft unterstellt wird.

Schlüsselwörter Einstellungen · Ökonomische Ungleichheit · Migration · Diversität · SOEP-Innovations-Stichprobe

\title{
The three arenas of new conflicts over inequality. An analysis of the relation between social structural positions and attitudes towards redistribution, migration and sexual diversity
}

\begin{abstract}
Many Western societies debate the question of redistribution with revived interest. However, the debates on identity politics and migration brought further social conflicts to the forefront. Academic as well as journalistic contributions often assume a two-camp polarisation in which groups with higher social status endorse migration and diversity against the opposition of groups with lower social status. In this view, the constellation of these new conflicts is at odds with classic conflicts over redistribution, in which higher social status groups typically show a greater tolerance of inequality compared to lower social status groups. The paper develops a conceptual framework that goes beyond the dominant two-camp polarisation theory and systematically distinguishes three dimensions of inequality conflicts and attitudes: firstly, classical up-down inequalities referring to economic redistribution, secondly, us-them inequalities relating to societal recognition of diversity, and thirdly, in-out inequalities concerning openness toward immigrants. Based on the SOEP Innovation Sample 2017, it is demonstrated that these attitudes constitute relatively independent dimensions. Compared to higher social groups, lower social status groups are more critical of economic inequality, while the two new conflicts indicate a reverse picture. However, only attitudes toward migration are substantially polarized, whereas little evidence can be found that points toward the formation of the two distinct cleavages that are frequently assumed in public discourse.
\end{abstract}

Keywords Attitudes · Economic inequality · Migration · Diversity · SOEP Innovation Sample

\section{Les trois arènes des nouveaux conflits liés aux inégalités. Une définition des coordonnées sociostructurelles des attitudes envers la redistribution, l'immigration et la diversité sexuelle}

Résumé La question de la répartition des richesses fait un retour en force dans de nombreuses sociétés occidentales. Avec le débat autour de la politique identitaire et de l'immigration d'autres conflits sont cependant venus occuper le devant de la scène de sorte que les inégalités matérielles ne concentrent plus toute l'attention. Dans les débats scientifiques et intellectuels, il est souvent question de la formation 
de nouveaux clivages entre les groupes privilégiés, qui se prononceraient en faveur de l'immigration et de la reconnaissance de la diversité, et les couches sociales plus défavorisées qui y seraient opposées. Ce conflit irait ainsi à rebours du conflit classique au sujet de la répartition des richesses dans lequel les occupants des positions sociales supérieures présentent une plus grande tolérance à l'égard de l'inégalité que les occupants des positions sociales inférieures plus critiques. Cet article développe un cadre conceptuel qui dépasse cette théorie du clivage en établissant une distinction systématique entre trois dimensions des conflits et des attitudes en matière d'inégalités : (1) les inégalités classiques haut-bas portant sur la répartition des ressources économiques, (2) les inégalités nous-eux comprenant la reconnaissance sociale de la diversité et (3) les inégalités intérieur-extérieur tournant autour du degré d'ouverture vis-à-vis des populations immigrées. Sur la base de l'échantillon « innovation » du panel socioéconomique (SOEP) allemand de l'année 2017, on peut montrer que ces attitudes constituent des dimensions relativement indépendantes. En matière de répartition des ressources économiques, les occupants des positions sociales inférieures sont plus critiques envers l'inégalité que les occupants des positions sociales supérieures ; concernant les deux sujets de conflits plus récents la situation est exactement inverse. Cependant, on ne constate une véritable polarisation des attitudes qu'en matière d'immigration. Dans les autres domaines, il existe peu d'indices de la formation de clivages aussi marqués que le prétendent la littérature et le débat public.

Mots-clés Attitudes · Inégalités économiques - Immigration · Diversité · Échantillon « innovation » du panel socioéconomique (SOEP) allemand

\section{Einleitung}

Betrachtet man die aktuellen Ungleichheitskonflikte in westlichen Gesellschaften, dann ist die Lage einigermaßen unübersichtlich. Neben dem vertikalen, ökonomisch geprägten Verteilungskonflikt, der, nach einer Zeit der weitgehenden Befriedung in der Nachkriegszeit, spätestens seit den 1980er-Jahren wieder an Aufmerksamkeit und Schärfe gewinnt, sind in der jüngsten Vergangenheit auch neue Spannungen in den Vordergrund getreten, die um Fragen der Migration und der Anerkennung nichtdominanter Identitäts- und Lebensformen kreisen (Foroutan 2019). Mit dieser Pluralisierung des Konfliktgeschehens scheint auch die Zuordnung gesellschaftlicher Gruppen zu Umverteilungs-, Integrations- und Anerkennungsfragen weniger eindeutig geworden zu sein. Prominente Gegenwartsdiagnosen sehen einerseits die akademische Mittel- und Oberschicht als treibende Kraft, wenn es um die Anerkennung sexueller Diversität und den Abbau ethnischer Diskriminierung geht; gleichzeitig werden gerade diese Schichten aber auch als die sozialstrukturellen Trägerschichten der neoliberalen Umgestaltung der sozialen Sicherungssysteme angesehen (z. B. Fraser 2017). Andererseits wird betont, dass in den unteren Statuslagen umverteilungsaffine Haltungen in Bezug auf ökonomische Ungleichheit durchaus mit rassistischen und homophoben Einstellungen Hand in Hand gehen können (z. B. Eribon 2016). 
Trotz anhaltender wissenschaftlicher und politischer Diskussionen über die Erweiterung und Mehrdimensionalität der gegenwärtigen Ungleichheitskonflikte sowie der damit einhergehenden Formierung sozialstruktureller Lager gibt es kaum Studien, welche Einstellungen in den drei wichtigen Konfliktdimensionen gleichzeitig und auf der Basis desselben Datensatzes analysieren. Dies wird im vorliegenden Aufsatz für Deutschland in Angriff genommen. Ganz konkret geht der Aufsatz der Frage nach, inwieweit die Einstellungen zu den drei Konfliktthemen ,ökonomische Umverteilung“, „Migration“" und ,sexuelle Diversität“ miteinander in Verbindung stehen und welche Positionen unterschiedliche sozialstrukturelle Gruppen diesbezüglich einnehmen. Nachfolgend wird zunächst der theoretische Rahmen skizziert (Abschnitt 2), bevor der Forschungsstand resümiert wird (Abschnitt 3) und die verwendeten Daten vorgestellt werden (Abschnitt 4). Unsere Datenbasis bildet die Innovations-Stichprobe des Sozio-oekonomischen Panels aus dem Jahr 2017. Diese Erhebung beinhaltete einen Befragungsschwerpunkt zu Einstellungen zu Umverteilung, Migration und sexueller Diversität und kann hier erstmals ausgewertet werden. Unsere Befunde werden schließlich im fünften Abschnitt präsentiert, bevor sie im sechsten Abschnitt zusammengefasst und auf ihre gesellschaftspolitischen Implikationen hin diskutiert werden.

Unsere Analysen zeigen, dass es sich bei den Haltungen zu den Themen ,ökonomische Umverteilung“, „Migration“ und ,sexuelle Diversität“ um relativ eigenständige Einstellungsdimensionen handelt. Zudem finden wir systematische Unterschiede zwischen den gesellschaftlichen Statuspositionen: Die oberen Statuslagen erweisen sich einerseits als ungleichheitsaffirmativer im Hinblick auf ökonomische Ungleichheiten, andererseits aber als anerkennungsbereiter als die unteren Lagen, wenn es um die „,neueren“ Konfliktthemen geht. Beim ,klassischen“ Thema der ökonomischen Ungleichheit sind die unteren Statuslagen am kritischsten positioniert. Allerdings sind die entsprechenden Einstellungsdifferenzen nur bei Fragen der Migration stärker konturiert und deuten auf eine Polarisierung hin. Demgegenüber existiert in allen Statusgruppen eine hohe Bereitschaft, sexuell diverse Identitäts- und Lebensformen anzuerkennen. Auch bei Fragen der Einkommensverteilung wünschen sich alle Statusgruppen eher einen Abbau bestehender Ungleichheiten.

\section{Theoretischer Rahmen}

Wie bereits betont, ist die Ungleichheitsfrage zu einer vertrackten Sache geworden. Das zeigt sich schon daran, dass, wenn von Ungleichheit die Rede ist, sehr unterschiedliche Dinge darunter verstanden werden: mal wird der Begriff vor allem auf die vertikale Ungleichverteilung materieller Güter bezogen, mal auf Fragen der Diskriminierung und Benachteiligung wie im Falle der Ungleichheit der Geschlechter oder des Rassismus, mal auf Fragen der Teilhabe und Inklusion wie im Hinblick auf Migration. In der Sprache der traditionellen Ungleichheitsforschung hätte man gesagt, dass zu den vertikalen die horizontalen Ungleichheiten hinzutreten, obwohl dies missverständlich ist, da diese gleichfalls mit ungleichen Lebenschancen und Lebensbedingungen einhergehen. Das Signum der Klassengesellschaft waren materielle Umverteilungskämpfe, während im Kontext der nunmehr politisierten 
Ungleichheiten oft Anerkennungskämpfe - beispielsweise um Minderheitenrechte, sexuelle Identität oder genderspezifische Ungerechtigkeiten - geführt werden. Die Agenda der sogenannten ,Identitätspolitik“ fußt einerseits auf einer wachsenden Sensibilität gegenüber Formen der Diskriminierung, andererseits geht es um Forderungen nach der Anerkennung diverser und nichtdominanter Identitäts- und Lebensformen, wobei oftmals gruppenspezifische Positionierungen, Merkmale und Identitäten von Gewicht sind. Teil der Debatte ist, ob Umverteilung und Anerkennung jeweils einen anderen Aspekt der Ungleichheits- und Gerechtigkeitskonflikte aktualisieren oder diese unauflösbar miteinander verbunden sind. In der normativen Theorie ist entsprechend diskutiert worden, inwieweit beide Achsen in einen gemeinsamen Bezugsrahmen gestellt werden können und sich jeweils auf die Zentralperspektive der Gerechtigkeit der Statusordnung beziehen lassen (siehe Fraser und Honneth 2003).

In zahlreichen jüngeren Publikationen ist immer wieder die These vertreten worden, dass anerkennungsorientierte politische Strömungen mit identitätspolitischer Ausrichtung die ,klassischen“ Ungleichheiten vernachlässigt hätten - sie seien ,klassenblind“ (Fraser 2017; Fukuyama 2018; Heisterhagen 2018; Lilla 2017a). Kritisch wird beispielsweise eingewandt, die identitätspolitische Programmatik betreibe einen Differenzkult (statt das Gemeinsame zu suchen) und sorge für eine Verfestigung kultureller Partikularismen, sodass der Blick auf die sozioökonomischen Ungleichheiten verstellt würde. Das symbolpolitische „Kulturtheater“ oder eine „Vielfaltseuphorie“ hätten nach und nach ökonomische Verteilungsfragen von der Agenda verdrängt (zu dieser Kritik siehe zusammenfassend van Dyk 2019). Mitunter wird die starke Hervorhebung von Identitätspolitik geradezu als politisches Dilemma der progressiven Linken beschrieben, die ihr eigentliches Brot-und-Butter-Thema, nämlich die Vertretung der Interessen materiell benachteiligter Gruppen, vernachlässigt hätten, wodurch ihnen auch die Unterstützung der arbeitenden Schichten weggebrochen sei (Fraser 2017). Linke Politik, die sich eigentlich um die Belange der sozial Schwachen kümmern solle, vertrete nunmehr immer weniger deren Interessen (Goodhart 2004), wohingegen Identitätspolitik zuvorderst ein Thema der Bessergestellten und der Hochgebildeten sei (Lilla 2017b; Stegemann 2017). ${ }^{1}$ Diese These gipfelt in dem Vorwurf, ,die da oben“ setzten sich nun vor allem für neue Lebensformen, gleichgeschlechtliche Ehen, Gender-Mainstreaming und Multikulturalismus ein und seien daher „selbstgefällig, behäbig und taub gegen die ,da unten“ geworden“ (Merkel 2017a, S. 56).

Auch zurückhaltender auftretende und differenziertere sozialstrukturelle Überlegungen zur Aufteilung der Mittelschicht in eine höher stehende „neue Mittelklasse“ - also vor allem die in der Wissensökonomie lokalisierte „Akademikerklasse“ - und eine ökonomisch und kulturell schwächere ,,alte Mittelklasse“, die noch eng mit den industriegesellschaftlichen Reproduktionsformen verbunden ist, legen ein ähnliches

\footnotetext{
1 Diesem Vorwurf ließe sich allerdings entgegnen, dass die Fragen der Anerkennung auch für die unteren Schichten relevant sein könnten. Auch der Mitarbeiter der Gebäudereinigung kann einen homosexuellen Sohn haben, oder seine Tochter hat einen Freund mit Migrationshintergrund. So gesehen könnte die sogenannte Identitätspolitik tatsächlich eine Angelegenheit aller Schichten (und nicht nur der kulturell und ökonomisch Privilegierten) sein.
} 
Muster nahe (Reckwitz 2019, S. 85 ff.; Reckwitz 2017). Hier sind die „,neuen Mittelklassen“ die wesentlichen Träger des Kosmopolitismus - verstanden als spezifisches Bündel von Einstellungen, etwa einer größeren Offenheit gegenüber Migration und der Vielfalt der Lebensformen sowie der Bejahung von Diversität und Gleichberechtigung -, während die ,alte Mittelklasse“ (zusammen mit der Unterklasse) tradierte Muster der Ordnungsbildung, Abgrenzung und Konformität bevorzuge. Geht es hier vor allem um die Analyse sozialstruktureller Wandlungsprozesse, so wird andernorts die Entgegensetzung der ,,akademisch-kosmopolitischen Mittelschicht“ und der kulturell „Deklassierten“ mitunter zum zentralen gesellschaftspolitischen Kampffeld erklärt, wobei in dieser Beschreibung die konservativen Fraktionen der oberen Schichten zu Alliierten der „Abgehängten“ werden (Koppetsch 2019, S. 123 ff.). ${ }^{2}$ Dabei stehen nun nicht mehr graduelle und sozialstrukturell grundierte Unterschiede im Mittelpunkt, sondern es wird die These vertreten, dass die ,akademischkosmopolitische Ober- und Mittelschicht" mit ihrem Habitus, ihren Einstellungen und soziokulturellen Praktiken eine rechtspopulistische Reaktion, unter anderem getragen von den ,zurückgefallenen Fraktionen der traditionellen Mittelschicht“ und den ,,prekären“ Verlierern“, geradezu provoziere (ebd., S. 221). So komme es aufgrund der kulturellen Hegemonie der Kosmopoliten aus der Mittel- und Oberschicht zu einer spezifischen Reaktanz der Deklassierten, die sich beispielsweise in der Geringschätzung bis Herabwürdigung wichtiger ,Symbole kosmopolitischer Gesinnung“ wie etwa „Multi-Kulti“ oder Transgender zeige (ebd., S. 122). ${ }^{3}$ Kurz: Jene mit kosmopolitischer Gesinnung seien mitverantwortlich für die rechtspopulistische Gegenwehr der „Unterlegenen“ und „,kulturell ,Dominierten““.

Jenseits solcher gegenwartsdiagnostischer Argumentationsfiguren kann man für den Zugriff auf neuere Konfliktdynamiken auch an etwas ältere Vorläufer im Feld der politischen Soziologie und der Politikwissenschaften anschließen, wobei damals vor allem die Analyse der unteren Schichten und der Arbeiterklasse im Vordergrund stand. So formulierte Seymor Martin Lipset bereits Ende der 1950er-Jahre seine empirisch unterfütterte These des ,working-class authoritarianism“ (Lipset 1959a, 1959b): Entgegen der Hoffnung vieler linker Intellektueller erwiesen sich die unteren Statuslagen selten als treibende Kraft für eine Ausweitung von Freiheit, Gleichheit und sozialem Fortschritt. Diese Gruppen mögen zwar häufig für ökonomische Umverteilung eintreten, gleichzeitig zeigen sie sich aber - aufgrund von isolierten Arbeitsplätzen, ökonomischer Unsicherheit und autoritären Erziehungsstilen - besonders intolerant bzw. zurückhaltend in Fragen der gesellschaftlichen Liberalisierung (Akzeptanz nichtdominanter Lebensformen, Gleichstellung ethnischer Minoritäten, Abbau von Diskriminierung). Bereits in den 1990er-Jahren findet sich zudem die These der Pluralisierung gesellschaftlicher Konfliktfelder bei

\footnotetext{
2 Das Buch Gesellschaft des Zorns (2019) von Cornelia Koppetsch ist aufgrund von Plagiaten und wissenschaftlichen Mängeln nicht mehr erhältlich; wir beziehen uns aber dennoch auf eine zentrale Argumentationsfigur des Buchs, weil diese Teil der akademischen und öffentlichen Diskussion geworden ist.

3 Diese „Reaktionsthese“ harrt aus unserer Sicht bislang der empirischen Verifizierung. In der genannten Arbeit von Koppetsch ist sie, soweit man erkennen kann, den Selbsterzählungen von AfD-Mitgliedern entnommen, nicht einer Untersuchung der Wählerschaften oder einer longitudinalen Beobachtung. Es wäre eine methodische Herausforderung, einen solchen kausalen Nexus nicht nur zu behaupten, sondern auch zu belegen.
} 
Herbert Kitschelt (1994, 2004): Angestoßen von Prozessen der Bildungsexpansion, Postindustrialisierung, Wohlfahrtsstaatsexpansion und Frauenemanzipation habe eine soziokulturelle Konfliktachse an Bedeutung gewonnen, die relativ losgelöst vom Konflikt um ökonomische Ressourcen ist. Durch diese Auffächerung des Konfliktgeschehens würden sich nicht nur große Teile der Bevölkerung neu orientieren, auch Parteien gerieten unter einen zunehmenden Re-Positionierungsdruck. Vergleicht man diese älteren Positionen mit den neueren Argumentationslinien, zeigt sich eine starke Ähnlichkeit in den Grundprämissen (plurale Konflikte, divergierende Positionen) wie auch in den problematischen Annahmen (es wird die Existenz von lediglich zwei Konfliktdimensionen behauptet, und es werden relativ starke Polarisierungen angenommen).

Es ist bemerkenswert, wie selten die nunmehr ausufernden und zuweilen aufgeregt geführten Debatten explizit und systematisch auf diese älteren Thesen Bezug nehmen, und es ist geradezu irritierend, dass sie fast gänzlich ohne Rückgriff auf Empirie auskommen, obwohl die Kernargumente auf starken empirischen Aussagen fußen. Im vorliegenden Aufsatz wollen wir deshalb das Thema empirisch wenden, indem wir uns schlicht die Frage stellen: Was ist dran an dieser Behauptung der Existenz zweier klar konturierter, mitunter sogar antipodisch verstandener Lager, die sich nicht nur weltanschaulich, sondern auch sozialstrukturell klar voneinander unterscheiden sollten? Wir fragen einerseits nach der sozialstrukturellen Basis und Profilierung dieser Ungleichheitsthemen sowie andererseits danach, ob die Unterscheidung zwischen ökonomischer Ungleichheit und Identitätspolitik trägt und trennscharf ist. Aufbauend auf Mau (2016) und Schimank (2018) wollen wir statt zwei Lager drei Arenen sozialer Ungleichheitskonflikte unterscheiden, die wir - zugegebenermaßen plakativ - als Oben-Unten-Ungleichheiten, Innen-AußenUngleichheiten und Wir-Sie-Ungleichheiten bezeichnen. ${ }^{4}$ Diese Unterscheidung, die zunächst heuristisch gemeint ist und dann einer empirischen Prüfung zugeführt wird, soll helfen, die Anordnung der Einstellungen zu wichtigen Fragen von Ungleichheit und Anerkennung auszuloten und unterschiedliche Dimensionen genauer zu differenzieren. Darauf aufbauend wird geprüft, ob wir in der Zustimmung zu diesen Themen tatsächlich einen sozialen Gradienten finden und, falls ja, ob er für die Themenkomplexe gleichgerichtet ist oder unterschiedlich ausfällt. Sind diese Themen in der Tat sozial divisiv, wie oft suggeriert wird? Stehen sie für sozialstrukturell klar verortbare Spaltungslinien? Doch zunächst zur Heuristik unterschiedlicher Ungleichheitsaspekte.

Mit Oben-Unten-Ungleichheiten bezeichnen wir die klassischen vertikalen Ungleichheiten hinsichtlich materieller Ausstattung, Einkommen und Vermögen. Die Oben-Unten-Unterscheidung bezieht sich in der Regel auf das sozioökonomische Gefälle innerhalb einer Gesellschaft, das durch die primäre und sekundäre Vertei-

\footnotetext{
${ }^{4}$ Eine vierte, sich in jüngerer Zeit herausbildende Achse könnte man die „Heute-Morgen-Ungleichheit“ nennen, die sich um die ökologische Frage und das Thema der Nachhaltigkeit dreht. Konkret geht es darum, inwieweit der gegenwärtige Ressourcenverbrauch und Konsum die Lebenschancen zukünftiger Generationen beeinträchtigt - im Kern ein Ungleichheitsthema. Die wohl stärkste Manifestation der Politisierung dieser Konfliktachse kann man in der Fridays-for-Future-Bewegung sehen. Aktuelle Befunde weisen darauf hin, dass das Thema Ökologie und Nachhaltigkeit besonders stark im Fokus der oberen Einkommensund Bildungsgruppen steht (Otto und Gugushvili 2020).
} 
lungspolitik adressiert wird, die zum Ziel hat, markterzeugte materielle Ungleichheiten $\mathrm{zu}$ begrenzen oder zu kompensieren. Es geht - pointiert formuliert - bei dieser Dimension um Klasse und Umverteilung, letztlich auch um Klassenkonflikte. $\mathrm{Zu}$ dieser Ungleichheitsachse gehörte traditionell die Unterscheidung zwischen Parteien im linken Spektrum, die eher umverteilungsaffin sind, und den eher marktorientierten wirtschaftsliberalen und konservativen Kräften auf der rechten Seite des Parteienspektrums (wobei diese Rechts-Links-Unterscheidung durch sozialpolitische Programmelemente im rechten Spektrum auch aufgebrochen wird). Befriedet, aber nicht aufgelöst wurde dieser Konflikt durch den institutionalisierten Klassenkompromiss, der im Ausbau sozialstaatlicher Institutionen, progressiver Besteuerung und einem Kranz an sozialen Rechten seinen Ausdruck fand. Dieser sollte ein Gegengewicht zur markterzeugten Ungleichheit bilden und diese auf ein gesellschaftlich akzeptables Maß reduzieren. Allerdings zeigt sich auch in der historischen Betrachtung, dass sich die Vermögens- und Einkommensungleichheiten vor allem in den Dekaden nach dem Zweiten Weltkrieg verringerten, dann aber Ende der 1970er- und in den 1980er-Jahren in vielen westlichen Ländern ein Umkehrtrend - zuweilen ,the great U-turn“ genannt (Alderson und Nielsen 2002) - einsetzte. Die Vermögens- und Einkommensungleichheiten nahmen sukzessive wieder zu, vor allem der Abstand zwischen den mittleren Lagen und den Spitzengruppen vergrößerte sich (Milanović 2016; OECD 2018; Piketty 2014). Im Hinblick auf den langfristigen Trend gilt dies auch für Deutschland. ${ }^{5}$ Auch in der Wahrnehmung der Bevölkerung stellt die vertikale Ungleichheit - verstanden als Reichtums- und Einkommensverteilung ein wichtiges Thema dar, und die Aufspreizung der Ungleichheitsschere zieht gesellschaftliche Kritik auf sich (Mau und Heuer 2016). In der Literatur ist vielfach belegt, dass die Kritik an Ungleichheit und die Befürwortung der Umverteilung mit der sozialstrukturellen Position systematisch variiert. Je niedriger die soziale Lage, desto stärker dominiert eine kritische Sicht auf Ungleichheit und desto größer ist das Interesse an einer umverteilenden Politik und der Begrenzung von Ungleichheit (stellvertretend für etliche andere: Svallfors 2006).

Der zweite Ungleichheitskomplex, der von uns als Dimension der Innen-AußenUngleichheiten bezeichnet wird, hat im Zuge der Globalisierung an Relevanz gewonnen, denn erst mit der forcierten Öffnung des nationalgesellschaftlichen Containers wurden die Konflikte um Öffnung und Schließung zur wichtigen gesellschaftlichen Konfliktlinie. Dabei sind es die ineinandergreifenden Prozesse von Globalisierung, Transnationalisierung und Europäisierung, die das einstmals recht abgeschlossene Gesellschaftsmodell herausfordern und das Thema politisierbar machen (Mau 2007). Von den Politikwissenschaften ist empirisch ausgeleuchtet worden, dass damit auch neue parteipolitische Konfliktordnungen entstanden sind, die das klassische, auf Umverteilung ausgerichtete Links-Rechts-Schema unterlaufen und die sich zwischen einem universalistischen und auf Offenheit setzenden Pol und einem eher auf

\footnotetext{
5 Auch wenn man keineswegs von einem linearen und kontinuierlichen Trend sprechen kann, kam es in Deutschland doch zu einer stärkeren Polarisierung der Einkommen (Grabka und Goebel 2018) und zur Konzentration von Vermögen und Erbschaften (Fratzscher 2016; Grabka und Westermeier 2014). Zugleich kam es im unteren Bereich der Sozialstruktur zur Verfestigung von Armut (Groh-Samberg 2014; GrohSamberg und Hertel 2015); ebenso sind Blockaden intergenerationeller Mobilität erkennbar (OECD 2018).
} 
nationalgesellschaftliche Schließung setzenden Pol aufspannen (Kriesi et al. 2008; de Wilde et al. 2019). Oft wird diese Konfliktlinie als Spannung zwischen Kosmopolitismus und Kommunitarismus oder zwischen „Globalisten“ und „Nativisten“ beschrieben (Koppetsch 2019; Merkel 2017a, b) - es geht um einen „Kampf über Grenzen“ (Zürn 2020, S. 165). ${ }^{6}$ Dabei dürften das Migrationsthema, die Offenheit und Geschlossenheit von Grenzen und die Frage des Zugangs von Migrantinnen und Migranten zu öffentlichen Leistungen eine herausgehobene Rolle spielen. Diese Konfliktlinie ist wahlweise als kultureller Backlash gegen den kosmopolitischen Linksliberalismus, als Reaktion auf ökonomische Unsicherheit und als Konsequenz von Veränderungen des politischen Systems und den Aufstieg internationaler Institutionen interpretiert worden, wobei jeweils die unteren und einfacheren Schichten stärker kommunitaristisch orientiert sein sollten (Norris und Inglehart 2019; Zürn 2020). Auf der Einstellungsebene könnte man von ,apertistischen“ Haltungen (so ein Begriff von Reckwitz 2017) auf der einen und „demarkationistischen“ Haltungen auf der anderen Seite sprechen (Schimank 2018, S. 230). Der Demarkationismus drückt sich im Ressentiment gegenüber Zuwanderung, protektionistischen SchlieBungsinteressen oder ethno-nationalistischen Vorstellungen von Gemeinschaft aus (Kraemer 2018), der Apertismus spiegelbildlich in einer Befürwortung der Öffnung des nationalgesellschaftlichen Raums und einer Inklusionsbereitschaft gegenüber Migrantinnen und Migranten. Diese Unterscheidung ist wiederum nicht auf Wertefragen - kulturelle Freiheitgewinne und Bereicherung durch Vielfalt versus kulturelle Irritationen und „Diversitätsstress“ - beschränkt, sondern hat auch eine ökonomische Dimension: Schließungs- und Öffnungsinteressen hängen auch davon $\mathrm{ab}$, inwieweit globale Marktintegration und ethnischer Wettbewerb als bedrohlich wahrgenommen werden - das wäre das ,bad opening“ (Schimank 2018, S. 230) oder inwieweit mit der Öffnung des nationalgesellschaftlichen Raums Marktchancen verbunden werden (zu den Effekten ökonomischer und sozialer Globalisierung auf unterschiedliche Statusgruppen siehe Mewes und Mau 2013). Aus der genannten Literatur lässt sich die These destillieren, dass es vor allem die oberen Schichten sein sollten, die für Öffnungsprozesse stehen, während die unteren eher für Schließung eintreten sollten, wobei wir diese Annahme anhand der Zuwanderungsfrage in den Blick nehmen wollen.

Drittens treten identitätspolitische Fragen ${ }^{7}$ im engeren Sinne hervor, die wir als Wir-Sie-Ungleichheiten beschreiben wollen. Ihr Treibstoff sind Diversität, Diskriminierungserfahrungen, Anerkennungsbestrebungen und Autonomieansprüche sozialer Gruppen, die in einer Gesellschaft leben (das unterscheidet sie zumindest analy-

\footnotetext{
${ }^{6}$ Mitunter werden weitere (kulturliberale) Themen wie Klimawandel, Individualismus, Geschlechterfragen oder die Gleichstellung Homosexueller gleichermaßen zum Bestandteil dieses Einstellungssyndroms erklärt. Wolfgang Merkel (2017a, S. 53) schreibt etwa: „Kosmopoliten priorisieren individuelle Rechte, offene Grenzen, liberale Zuwanderung, erleichterte Einbürgerung, kulturellen Pluralismus sowie eine globale Verantwortung für universell gültige Menschenrechte und den Umweltschutz." Weitere Bezüge dazu finden sich bei Koppetsch (2019).

7 Wobei einzuräumen ist, dass es sich bei dem Begriff der „Identitätspolitik“ um einen ungenauen und schwammigen Begriff handelt, der zuweilen als ,leerer Signifikant“ herhalten muss, weil sehr unterschiedliche Dinge darunter subsumiert werden (etwa Antidiskriminierung, Toleranz, Diversität, Anerkennung, Selbstbestimmung etc.) (siehe dazu van Dyk und Graefe 2019).
} 
tisch von den Innen-Außen-Ungleichheiten). Im Hinblick auf Geschlecht, Minderheitenstatus, Hautfarbe, sexuelle Identität oder nichtnormierte Lebensweisen sollen Formen der Diskriminierung, Marginalisierung und Schlechterstellung vermieden werden (diese Aspekte werden allerdings oft mit der Frage der offenen Grenzen zusammengeführt, siehe Norris und Inglehart 2019). Konkret geht es beispielsweise um Gender-Mainstreaming, die rechtliche Gleichstellung homosexueller Partnerschaften, Geschlechtsidentitäten jenseits von Mann und Frau, die gesellschaftliche Akzeptanz von sozialer Diversität, das Heraustreten aus einer Position der Marginalität und vieles weitere mehr. Die Gegenposition ist die des Hochhaltens der Heteronormativität, der Kritik des „Diversitätskults“, der Homophobie sowie die Position des Anti-Genderismus (Hark und Villa 2015). ${ }^{8}$ Statt um Klasse und Umverteilung oder Schließung und Zugang geht es hier - so könnte man begrifflich pointieren - nunmehr um Identität und Anerkennung. Auch auf dieses Konfliktfeld lässt sich eine ungleichheitssoziologische Perspektive richten, indem man fragt, welche sozialstrukturellen Gruppen eine größere Affinität zu diesem Thema aufweisen und welche eine geringere bzw. welche Gruppen sogar in Gegnerschaft zu den emanzipatorischen oder soziokulturell libertären Ansprüchen treten. Andreas Reckwitz (2017) sieht die neuen kulturellen Mittelklassen als Träger eines ,differenziellen Liberalismus“, der Diversität in sozialer, ethnischer, kultureller und lebensstilbezogener Hinsicht bejaht und deswegen auch gesellschaftlichen Normierungen kritisch gegenübersteht, während die alten (unteren) Mittelklassen und die unteren Schichten zu einem größeren Konformismus neigen. Das, so jedenfalls die These, sollte sie gegenüber der Pluralität neuer Lebensformen, neuen Ansprüchen auf soziale und geschlechtliche Identitäten, Anerkennungsforderungen der „Anderen“ oder gewachsenen Sensibilisierungen gegenüber Diskriminierung zurückhaltender und mitunter sogar ablehnend sein lassen. Empirische Arbeiten deuten darauf hin, dass soziokulturell libertäre Positionen stärker bei den bessergestellten Berufsgruppen und weniger bei den Produktionsarbeiterinnen und -arbeitern verbreitet sind (Oesch und Rennwald 2018; Svallfors 2006). Während es hier noch um graduelle Unterschiede geht, wird diese Spannung in zahlreichen publizistischen Interventionen zum Kulturkampf zugespitzt. Der Kampf gegen Wir-Sie-Ungleichheiten wird als elitärer Diskurs der Privilegierten angesehen, dem eine (ökonomische wie kulturelle) „Querfront der Verlierer" gegenübersteht (siehe etwa Koppetsch 2019). Die neue soziokulturelle Agenda (die auch die Sozialdemokratie erfasst habe) wird auch als ursächlich für die Hinwendung einfacher und arbeiterlicher Wählerschichten zu rechtspopulistischen Bewegungen gesehen (Heisterhagen 2018; Jörke und Heisterhagen 2017). Wäre diese These stichhaltig, dann sollte sich beispielsweise bei einer empirischen Beschäftigung mit den Einstellungen zur Homosexualität oder der Akzeptanz von Transgender-Identitäten ein sozialer Gradient zwischen den bessergestellten und den unteren Schichten ergeben.

Fassen wir die Ausgangsannahmen entlang der von uns unterschiedenen Ungleichheitsachsen zusammen: Ungleichheitskritik beim Thema der Oben-Unten-Un-

\footnotetext{
8 In der politischen Auseinandersetzung verbinden sich diese Positionen nicht selten mit rechtspopulistischen Mobilisierungsthemen wie völkischen Gemeinschaftsvorstellungen und Xenophobie; aus analytischen Gesichtspunkten trennen wir sie aber.
} 
Tab. 1 Postulierte Zusammenhänge zwischen Haltungen in Konfliktfeldern und sozialstruktureller Position (Quelle: Eigene Darstellung)

\begin{tabular}{lll}
\hline Konfliktfeld & Sozialstrukturelle Verortung der Haltungen \\
\hline Oben-Unten- & Affirmativ: & Kritisch: \\
Ungleichheit & Hohes Einkommen & Geringes Einkommen \\
& Hohe Bildung & Geringe Bildung \\
Innen-Außen- & Affirmativ: & Kritisch: \\
Ungleichheit & Geringes Einkommen & Hohes Einkommen \\
& Geringe Bildung & Hohe Bildung \\
Wir-Sie-Ungleichheit & Affirmativ: & Kritisch: \\
& Geringes Einkommen & Hohes Einkommen \\
& Geringe Bildung & Hohe Bildung \\
\hline
\end{tabular}

gleichheiten sollte vor allem im Fokus der unteren Schichten stehen, bei den InnenAußen-Ungleichheiten kann man demarkationistische Haltungen eher bei den unteren Statusgruppen vermuten und apertistische eher bei den oberen, bei den Wir-SieUngleichheiten nehmen wir entsprechend an, dass die Zustimmung für eine anerkennungsorientierte Identitätspolitik mit dem sozialen Status zunehmen sollte (siehe Tabelle 1). Wir wollen nun im Folgenden wissen, ob und wie diese Themen zusammenhängen, wie hoch jeweils die Zustimmung zu diesen Themenkomplexen ist und welche sozialstrukturellen Muster sie aufweist. Erst wenn sich jeweils starke sozialstrukturelle Gradienten in den Haltungen finden würden, könnte man von einer Cleavage im sozialstrukturellen Sinne sprechen.

\section{Forschungsstand}

Der Forschungsstand zu den uns interessierenden Ungleichheitseinstellungen ist relativ konsolidiert und lückenhaft zugleich: Während es zahlreiche Studien zu den einzelnen Themenbereichen gibt, existieren nur wenige Befunde, die mehrere Einstellungsdimensionen simultan betrachten. Im Folgenden skizzieren wir kurz die Befundlage zu den einzelnen Dimensionen.

Einstellungen zu ökonomischer Ungleichheit stellen ein klassisches Forschungsfeld der Ungleichheits- und Einstellungsforschung dar. In dieser Forschung wird häufig betont, dass sich Länder u.a. aufgrund kultureller Traditionen und institutioneller Strukturen darin unterscheiden, inwieweit die Bevölkerung bestehende ökonomische Ungleichheiten akzeptiert und als legitim anerkennt und inwieweit sie wohlfahrtsstaatliche Eingriffe fordert. Innerhalb der Länder gibt es typischerweise deutliche Einstellungsunterschiede zwischen sozialstrukturellen Gruppierungen, die sich als Reaktion auf gruppenspezifische soziale und ökonomische Lebenssituationen ergeben (Liebig und Wegener 1995). Dabei sind es insbesondere die unteren Klassenlagen, die besonders ungleichheitskritisch und umverteilungsaffin auftreten (Svallfors 2006). Auch für weitere Statusindikatoren (etwa Einkommen, Bildung und subjektiver Status) findet sich ein entsprechender sozialstruktureller Gradient in den Einstellungen (Fatke 2018; Fernández und Jaime-Castillo 2017; Mau 2003; Noll und Weick 2012; Roex et al. 2019; Steele 2015). Allerdings wurde auch gezeigt, 
dass sich ein solcher Gradient abschwächen kann, wenn die Ungleichheitskritik im Zeitverlauf bevölkerungsübergreifend zunimmt (Lux 2011).

Auch Einstellungen zu gesellschaftlicher Öffnung und Schließung sind inzwischen ein etabliertes Forschungsfeld, bei dem der Fokus auf Fragen der Zuwanderung und der Einbeziehung der Migrationsgruppen ohne Staatsbürgerschaft in die Sozialleistungssysteme liegt. Vielfältige Forschungen haben belegt, dass vor allem die unteren Statuslagen stärkere Schließungsinteressen haben, wobei in der Diskussion ist, ob eher kulturelle oder ökonomische Motive hierbei ausschlaggebend sind (z. B. Lengfeld und Dilger 2018; Mewes und Mau 2012, 2013). Bildung, Einkommen und Berufsposition haben sich als starke Determinanten migrationskritischer und wohlfahrtschauvinistischer Haltungen erwiesen (Davidov et al. 2014; Kunovich 2004; Mewes und Mau 2012; Semyonov et al. 2006). Der Einfluss dieser Faktoren wird dadurch erklärt, dass Personen, die sich in einer prekären sozioökonomischen Position befinden, Migranten eher als kulturelle Bedrohung oder Konkurrenten um knappe Ressourcen (etwa auf dem Arbeitsmarkt) wahrnehmen (Kunovich 2004; Raijman et al. 2003). Es wird jedoch auch betont, dass es sich hierbei um eine subjektiv empfundene Bedrohung handeln kann, der keine objektive Bedrohungslage entsprechen muss (Semyonov et al. 2004). ${ }^{9}$ Gruppen mit einem hohen (transnationalen) Humankapital werden hingegen eher den Gewinnern von Öffnungsprozessen zugerechnet (Teney et al. 2014; Gerhards et al. 2016); dementsprechend sehen sie die Globalisierung positiver, empfinden weniger „ethnischen Wettbewerb“, weisen eine größere Offenheit und Inklusionsbereitschaft auf und betonen die Bereicherung durch den Kontakt mit Zuwanderinnen und Zuwanderern (Mau 2007).

Die Literatur zu Einstellungen zu sexueller Diversität unterscheidet zwischen individuellen und kontextuellen Faktoren, um Einstellungsdifferenzen zu erklären (Adamaczyk und Liao 2019). Studien, die sich mit Einstellungen zu Homosexualität beschäftigen, deuten darauf hin, dass die Gesellschaften zwar im Durchschnitt liberaler geworden sind, aber weiterhin starke Unterschiede zwischen den Ländern bestehen (ebd., S. 409 ff.). Hinsichtlich statusbezogener und individueller Merkmale zeigt sich, dass Geschlecht, Einkommen, Bildung, Alter und Religion starke Prädiktoren sind. Insbesondere Frauen, Besserverdienende, Personen mit einer hohen Bildung, junge Personen und Individuen, die keiner konservativen religiösen Strömung angehören, sind offener gegenüber Homosexualität eingestellt (ebd., S. 412f.). Weitere Studien belegen, dass Arbeiter eine rigidere Sexualmoral besitzen und eine höhere Ablehnung gegenüber gleichgeschlechtlichen Beziehungen äußern als Personen aus höheren Schichten (Andersen und Fetner 2008; Svallfors 2006). ${ }^{10}$ Während bereits eine ganze Reihe von Studien zu Einstellungen zu Homosexualität vorliegt, ist dies hinsichtlich der Einstellungen gegenüber Transgender-Personen nicht gegeben. Zwar belegen Studien, dass Homo- und Transphobie stark korrelieren (Nagoshi

\footnotetext{
9 Berücksichtigt man weitere kontextuelle Faktoren, wie die Anzahl an Migranten in der Region oder die regionale Arbeitslosenquote, so deuten Studien darauf hin, dass eine hohe Anzahl von Migranten das Wählerpotenzial von rechtsradikalen Parteien tendenziell einschränkt, was für die sogenannte Kontakthypothese spricht, während eine hohe Arbeitslosenquote rechtsradikale Parteien eher stärkt (Teney 2012).

10 Empirische Analysen, die makrostrukturelle Faktoren einbeziehen, dokumentieren zudem, dass demokratische, wohlhabende und wenig religiöse Gesellschaften im Durchschnitt offener gegenüber sexuellen Minderheiten sind (Adamaczyk und Liao 2019).
} 
et al. 2008), aber es werden abseits von geschlechtsspezifischen Einstellungsunterschieden kaum weitere soziostrukturelle Merkmale berücksichtigt (ebd.; Tebbe und Moradi 2012).

Insgesamt deutet der Forschungsstand auf systematische Einstellungsunterschiede zwischen niedrigeren und höheren Schichten hin: Während Personen mit einer hohen Bildung und einem überdurchschnittlichen Einkommen tendenziell ungleichheitskritische Einstellungen bei den Innen-Außen-Ungleichheiten und den Wir-SieUngleichheiten aufzuweisen scheinen, sind sie hinsichtlich der Oben-Unten-Ungleichheiten eher affirmativ. Anders scheinen Gruppen positioniert, die sich durch eine geringe Bildung sowie ein niedriges Einkommen auszeichnen. Diese sind bezüglich der Oben-Unten-Ungleichheiten kritisch eingestellt, während sie weniger liberale bzw. offene Einstellungen gegenüber sexuellen Minderheiten und Zuwanderung aufweisen. ${ }^{11}$ Hierbei bestehen allerdings gravierende Unterschiede zwischen einzelnen Ländern, die in den entsprechenden Studien als kontextuelle Faktoren eingehen. Auch sind die Zusammenhänge zwischen Statusmerkmalen und Einstellungen nicht immer stark, sodass nicht notwendigerweise von einem sozialstrukturell polarisierten Konfliktfeld ausgegangen werden kann. Es bleibt zudem unklar, ob sich spezifische Einstellungen innerhalb eines Konfliktfeldes in den anderen Bereichen fortsetzen, wir also von übergreifenden, sozial ähnlich strukturierten oder gar zusammenhängenden Einstellungssyndromen ausgehen können. Im Folgenden nehmen wir eine sozialstrukturelle Kartierung der genannten Einstellungen gleichzeitig über die einzelnen Konfliktfelder vor, die es uns erlaubt, diese in einen direkten Zusammenhang zu bringen.

\section{Daten und Operationalisierungen}

Für unsere Analysen nutzen wir Daten der Innovations-Stichprobe des Sozio-oekonomischen Panels (SOEP-IS) aus dem Jahr 2017. Bei der SOEP-InnovationsStichprobe handelt es sich um eine repräsentative Erhebung der Wohnbevölkerung in Deutschland im Alter von 17 Jahren oder älter (Richter und Schupp 2015). Im Rahmen dieser Erhebung besteht die Möglichkeit, innovative Fragen zu aktuellen Themen zu platzieren, die in der Hauptbefragung des SOEP keinen Raum finden. Im Jahr 2017 konnten wir auf diesem Weg Fragen zu Ungleichheitseinstellungen integrieren, die ca. 1800 Untersuchungspersonen im Kontext von computerstützten persönlichen Interviews vorgelegt wurden (Subsample I2 und Subsample I4; Zweck und Glemser 2019). Konkret handelte es sich um folgende sechs Aussagen, zu denen die Interviewten ihre Zustimmung bzw. Ablehnung auf einer 7-stufigen Skala zum Ausdruck bringen konnten (die Skala reichte jeweils von ,stimme gar nicht zu“ bis

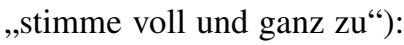

1. Die Einkommensunterschiede in Deutschland sind zu groß.

$11 \mathrm{Zu}$ ähnlichen Befunden kommt auch Floris Biskamp (2020), der jüngst auf seinem Blog die sozialstrukturelle Profilierung der Einstellungen zu Europäischer Integration, Migration, LGBT-Rechten und staatlicher Umverteilungspolitik auf Basis des European Social Survey untersucht hat. 
2. Es ist die Aufgabe des Staates, die Einkommensunterschiede zwischen den Leuten mit hohem Einkommen und solchen mit niedrigem Einkommen zu verringern.

3. Heutzutage gibt es in Deutschland zu viele Ausländer.

4. Personen ohne deutsche Staatsangehörigkeit, die legal nach Deutschland gekommen sind, sollten die gleichen Sozialleistungen erhalten wie deutsche Staatsangehörige.

5. Gleichgeschlechtliche Ehen sollten akzeptiert werden.

6. Es wäre gut für die Gesellschaft, wenn Transgender-Personen (Personen, die ihr biologisches Geschlecht geändert haben) als normal anerkannt werden. ${ }^{12}$

Aus konzeptioneller Sicht sollten sich die ersten beiden Aussagen auf die Dimension der Oben-Unten-Ungleichheit beziehen, die nächsten beiden auf die InnenAußen-Ungleichheit, und die zwei letzten Aussagen sollten die Wir-Sie-Ungleichheit abbilden. Die entsprechenden Zustimmungen bzw. Ablehnungen wurden von uns so rekodiert, dass höhere Werte ungleichheitskritische Haltungen zum Ausdruck bringen, d.h. Haltungen, nach denen die Einkommensungleichheit reduziert, sexuell diverse Identitäts- und Lebensformen anerkannt und eine stärkere Offenheit gegenüber Migration existieren sollte.

Wir können mit unseren Daten die drei aufgemachten Themenblöcke keinesfalls in Gänze abbilden, da hierzu umfangreichere und detaillierte Einstellungsbatterien notwendig wären. Die Integration solch erweiterter Einstellungsbatterien war im Rahmen der Erhebung der SOEP-Innovations-Stichprobe nicht möglich, und es existieren auch keine anderen Datensätze, mit denen alle drei von uns anvisierten Themenblöcke umfassend und differenziert abgebildet werden können. Trotz dieser Einschränkung gehen wir davon aus, dass die ausgewählten Items wichtige Aspekte der jeweiligen Issues erfassen. Zudem haben sich unsere Items bereits in anderen themenspezifischen empirischen Untersuchungen bewährt (Gerhards 2010; Heath und Tilley 2005; Pollack 2014; Svallfors 2006; Walch et al. 2012).

Als Indikatoren der sozialen Lage nutzen wir die Einkommensschicht und die Bildungsgruppe. Die Einkommensschicht basiert auf dem Nettoäquivalenzeinkommen, bei dem das Haushaltsnettoeinkommen mit der Haushaltsgröße und -zusammensetzung gewichtet wurde (neue OECD-Skala), um die mit einer wachsenden Haushaltsgröße verbundenen Einspareffekte zu berücksichtigen. Das so ermittelte Einkommen wurde in drei Schichten unterteilt: Unterschicht (weniger als 75 Prozent des medianen Nettoäquivalenzeinkommens), Mittelschicht (75 bis 150 Prozent) und Oberschicht (mehr als 150 Prozent). Bei der Bildung wurde der höchste erreichte allgemeinbildende Schulabschluss in drei Kategorien unterteilt: geringe Bildung (maximal Hauptschulabschluss), mittlere Bildung (Realschulabschluss) und hohe

\footnotetext{
12 Generell ist „Transgender“ ein Oberbegriff für jene Personen, die sich nicht mit ihrem angeborenen biologischen Geschlecht identifizieren, was nicht notwendigerweise den Wechsel des biologischen Geschlechts (Transition) beinhaltet (Berg-Weger 2016, S. 229). Bei dem vorliegenden Item verwenden wir eine enge Definition von Transgender, die eine solche Transition explizit voraussetzt. Dies schien aus unserer Sicht notwendig, um das Item für möglichst viele Untersuchungspersonen (mit unterschiedlichem Alter und Bildungshintergrund) verständlich zu halten. In der Folge ist der Anteil von Antwortverweigerungen zu diesem Item nur geringfügig höher als bei den anderen einbezogenen Items (ca. 5 Prozent versus 1-2 Prozent).
} 
Bildung (Abitur oder mehr). Einkommen und Bildung sind einschlägige Indikatoren, die die Schichtung der Statusordnung entlang der oben genannten Thesen gut erfassen können. Die bei Reckwitz (2017, 2019) wichtige Differenzierung zwischen neuer und alter Mittelklasse, die auch stark an Tätigkeitsfelder und Branchen gebunden ist, wird aber allenfalls mittelbar (und konzeptionell unvollständig) durch die Bildung einbezogen. Zusätzlich kontrollieren wir in unseren multivariaten Analysen für das Alter, den Familienstand, das Geschlecht, die Wohnregion und den Migrationsstatus. Eine Übersicht über die (Re-)Kodierung dieser Variablen sowie deren Verteilung findet sich in Tabelle $4 \mathrm{im}$ Anhang. Wir beziehen in unsere Analysen alle Fälle ein, die bei jedem der oben angeführten Einstellungsitems einen gültigen Wert aufweisen. Damit stehen uns 1641 Fälle zur Verfügung.

\section{Ergebnisse}

Betrachtet man die Ungleichheitseinstellungen der deutschen Bevölkerung im Jahr 2017, fällt auf, dass ungleichheitskritische Haltungen in Bezug auf die ökonomische Ressourcenverteilung sehr weit verbreitet sind (siehe Abbildung 1a, b): Ganze 86 Prozent stimmen der Aussage zu, dass die Einkommensunterschiede in Deutschland zu groß sind (Zustimmungswerte zwischen 5 und 7), während lediglich 14 Prozent hier eine andere Meinung haben (Zustimmungswerte zwischen 1 und 4). Gleichzeitig sehen etwa 71 Prozent der Bevölkerung den Staat in der Verantwortung, wenn es darum geht, diese Einkommensunterschiede zu verringern. Eine andere Verantwortungszuschreibung treffen lediglich 29 Prozent. Eine ähnlich eindeutige Tendenz zeigt sich auch bei der Anerkennung sexuell diverser Identitäts- und Lebensformen (siehe Abbildung 1c, d). Hier sind ca. 70 Prozent der Meinung, dass Transgender-Personen als normal anerkannt werden sollen, und ca. 72 Prozent befürworten gleichgeschlechtliche Ehen. Lediglich bei 30 bzw. 28 Prozent der Bevölkerung findet sich eine zurückhaltende bis ablehnende Haltung.

Während in Bezug auf die Einkommensverteilung und sexuelle Diversität also eine deutliche Mehrheit der Bevölkerung ungleichheitskritische bzw. anerkennungsbereite Haltungen aufweist, ergibt sich bei Fragen der Zuwanderung und des Status von Nicht-Staatsbürgern ein anderes Bild (siehe Abbildung 1e und f). Hier kommen exkludierende und ungleichheitsbejahende Einstellungen wesentlich häufiger vor als in den anderen beiden Dimensionen, und die Einstellungen sind insgesamt breiter über die Antwortskala gestreut. So lehnt nur eine Minderheit von ca. 32 Prozent der Bevölkerung die Aussage ab, dass zu viele Ausländer in Deutschland leben, während ganze 68 Prozent dieser Aussage zustimmen oder zu keiner klaren Einschätzung kommen. Zudem ist nur knapp die Hälfte der Bevölkerung der Meinung, dass Personen ohne deutsche Staatsangehörigkeit, die legal nach Deutschland gekommen sind, die gleichen Sozialleistungen erhalten sollten wie deutsche Staatsangehörige. Die andere Hälfte der Bevölkerung will solchen Migranten nur eingeschränkte Sozialrechte gewähren oder kommt diesbezüglich zu keiner eindeutigen Positionierung. Schon dieser erste Blick auf die univariaten Verteilungen der Zustimmungs- und Ablehnungswerte lässt also Zweifel daran aufkommen, dass in allen drei von uns betrachteten Ungleichheitsarenen eine ähnlich starke Polarisierung der Einstellungen 
a Die Einkommensunterschiede in Deutschland sind zu groß.

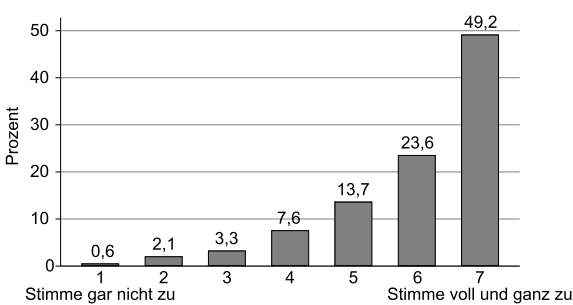

C Gleichgeschlechtliche Ehen sollten akzeptiert werden.

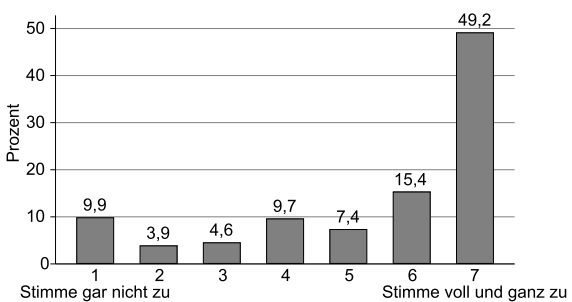

e

Heutzutage gibt es in Deutschland zu viele Ausländer.

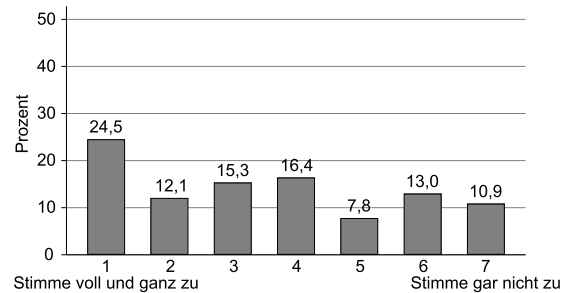

b Es ist die Aufgabe des Staates, die Einkommensunterschiede zwischen den Leuten mit hohem Einkommen und solchen mit niedrigem Einkommen zu verringern.

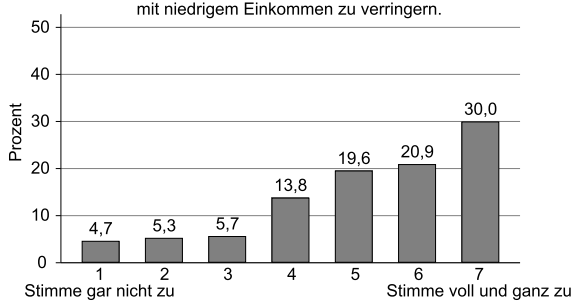

d Es wäre gut für die Gesellschaft wenn Transgender-Personen als normal anerkannt werden.

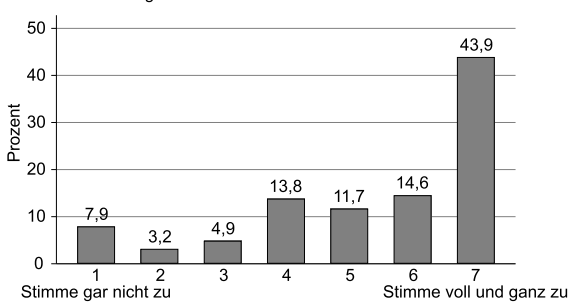

f Personen ohne deutsche Staatsangehörigkeit, die legal nach Deutschland gekommen sind, sollten die gleichen Sozialleistungen

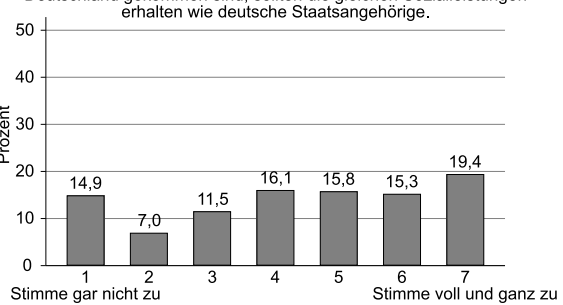

Abb. 1 Verteilung der Zustimmung bzw. Ablehnung zu den Einstellungsitems. (Quelle: SOEP-Innovations-Stichprobe 2017; eigene Berechnungen, gewichtet). Die numerischen Werte der Antwortskala von Teilgrafik 1e wurden von uns invertiert

vorliegt. Viel eher deuten unsere Befunde auf einen relativ breiten gesellschaftlichen Konsens bei Fragen der ökonomischen Ungleichheit und der Anerkennung sexueller Diversität bzw. geschlechtlicher Identiät hin. Beim Zuwanderungsthema sind die Einstellungen der Bevölkerung allerdings deutlich divergenter.

Nachdem wir einen ersten Überblick über die Zustimmungen bzw. Ablehnungen zu den einzelnen Aussagen gegeben haben, soll nun geprüft werden, ob die Haltungen zu diesen Aussagen auch empirisch der von uns getroffenen theoretischen Gruppierung entsprechen. Es geht nun also darum zu prüfen, ob es sich bei den Einstellungen zu Einkommensverteilung, sexueller Diversität und Zuwanderung tatsächlich um drei einzelne Einstellungsdimensionen handelt oder ob diese Einstellungen so eng miteinander verknüpft sind, dass sie nicht voneinander zu trennen sind, also bestimmte Haltungen in einer Dimension nahezu zwangsläufig auch mit 
Tab. 2 Explorative Faktorenanalyse, Faktorladungen und 95\%-Konfidenzintervalle (Quelle: SOEP-Innovations-Stichprobe 2017; eigene Berechnungen, ungewichtet)

\begin{tabular}{|c|c|c|c|c|c|c|c|}
\hline \multirow[t]{2}{*}{ Item } & \multicolumn{2}{|l|}{ Faktor 1} & \multicolumn{2}{|l|}{ Faktor 2} & \multicolumn{2}{|l|}{ Faktor 3} & \multirow{2}{*}{$\begin{array}{l}\text { Kommu- } \\
\text { nalität }\end{array}$} \\
\hline & Ladung & $95 \%-\mathrm{KI}$ & Ladung & $95 \%-\mathrm{KI}$ & Ladung & $95 \%-\mathrm{KI}$ & \\
\hline $\begin{array}{l}\text { Oben/ } \\
\text { Unten I }\end{array}$ & 0,04 & 0,$00 ; 0,09$ & 0,68 & 0,$60 ; 0,81$ & $-0,02$ & $-0,07 ; 0,02$ & 0,48 \\
\hline $\begin{array}{l}\text { Oben/ } \\
\text { Unten II }\end{array}$ & $-0,02$ & $-0,08 ; 0,07$ & 0,65 & 0,$53 ; 0,73$ & 0,02 & $-0,07 ; 0,09$ & 0,41 \\
\hline $\begin{array}{l}\text { Innen/ } \\
\text { Außen I }\end{array}$ & 0,04 & $-0,03 ; 0,14$ & $-0,15$ & $-0,21 ;-0,10$ & 0,57 & 0,$44 ; 0,64$ & 0,38 \\
\hline $\begin{array}{l}\text { Innen/ } \\
\text { Außen II }\end{array}$ & $-0,03$ & $-0,07 ; 0,02$ & 0,13 & 0,$09 ; 0,17$ & 0,60 & 0,$50 ; 0,77$ & 0,35 \\
\hline Wir/Sie I & 0,82 & 0,$76 ; 0,94$ & $-0,02$ & $-0,05 ; 0,01$ & $-0,03$ & $-0,07 ; 0,01$ & 0,65 \\
\hline Wir/Sie II & 0,79 & 0,$69 ; 0,85$ & 0,04 & 0,$00 ; 0,09$ & 0,04 & $-0,02 ; 0,13$ & 0,66 \\
\hline
\end{tabular}

Oben/Unten I: Die Einkommensunterschiede in Deutschland sind zu groß. Oben/Unten II: Es ist die Aufgabe des Staates, die Einkommensunterschiede zwischen den Leuten mit hohem Einkommen und solchen mit niedrigem Einkommen zu verringern. Innen/Außen I: Heutzutage gibt es in Deutschland zu viele Ausländer. Innen/Außen II: Personen ohne deutsche Staatsangehörigkeit, die legal nach Deutschland gekommen sind, sollten die gleichen Sozialleistungen erhalten wie deutsche Staatsangehörige. Wir/Sie I: Gleichgeschlechtliche Ehen sollten akzeptiert werden. Wir/Sie II: Es wäre gut für die Gesellschaft, wenn Transgender-Personen (Personen, die ihr biologisches Geschlecht geändert haben) als normal anerkannt werden.

bestimmten Haltungen in einer anderen Dimension einhergehen. Dann müsste man - wie oft in der Literatur angenommen - von einem einheitlichen Einstellungssyndrom und nicht von relativ eigenständigen Dimensionen ausgehen. Hierzu wurde von uns eine exploratorische Faktorenanalyse (EFA) durchgeführt, welche im Folgenden dargestellt wird. ${ }^{13}$ Das spezifische Vorgehen ist im Online-Anhang beschrieben.

Tabelle 2 zeigt, dass die Haltungen zur Einkommensverteilung, zur sexuellen Diversität und zur Migration nicht nur theoretisch, sondern auch empirisch drei abgrenzbare Dimensionen darstellen. Es zeigt sich ein eindeutiges Muster mit jeweils zwei Indikatoren, die stark auf einem Faktor laden. Zudem liegen nur vernachlässigbare Kreuzladungen zwischen den Faktoren und den einzelnen Items vor. Das bestätigt unsere Vorüberlegungen. Alle drei Einstellungskomplexe werden als eigene Faktoren ausgewiesen; ein einfacher Dualismus von Klassenpolitik versus Identitätspolitik bzw. Kosmopolitismus versus Kommunitarismus, der umfassende Einstellungssyndrome abbildet, zeigt sich bei der Einstellungsanalyse nicht. Allerdings sind die einzelnen Dimensionen auch nicht vollständig unabhängig, was sich an einer schwachen bzw. mittleren Korrelation zwischen den Dimensionen „Oben/ Unten“ und „Wir/Sie“ (0,23) sowie „Innen/Außen“ und „Wir/Sie“ $(0,49)$ zeigt.

Aufgrund dieser empirischen Struktur bilden wir im Folgenden Summenindizes für die drei Einstellungsdimensionen, in die jeweils die beiden von uns angedachten

\footnotetext{
${ }^{13}$ Die zusätzlich durchgeführten konfirmatorischen Faktorenanalysen (KFA) stützen die Ergebnisse der explorativen Faktorenanalyse. Aus Platzgründen präsentieren wir hier nur die Ergebnisse der EFA. Die Ergebnisse der KFA stehen auf Anfrage bei den Autoren zur Verfügung. Auf die Anwendung gruppierender Verfahren (wie bspw. Clusteranalyse oder latente Klassenanalyse) wurde verzichtet, da solche Verfahren zu kategorialen Zielvariablen führen, wir aber den Informationsreichtum der vorliegenden metrischen Variablen erhalten wollen.
} 

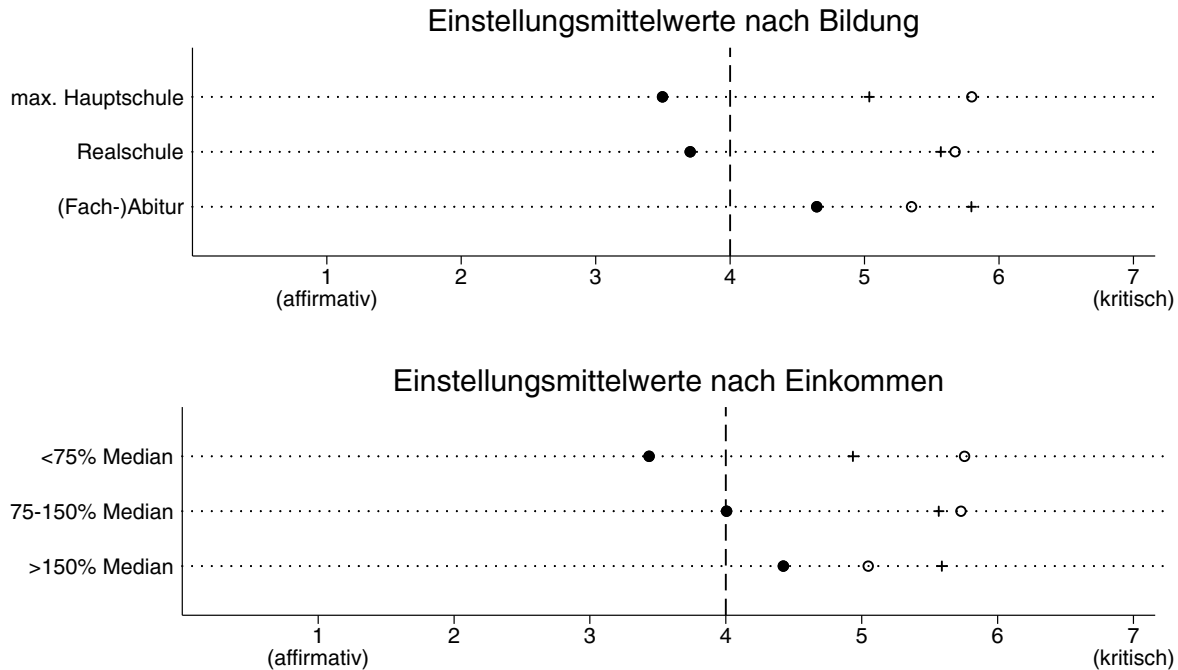

- Innen-Außen-Ungleichheit

+ Wir-Sie-Ungleichheit

○ Oben-Unten-Ungleichheit

Abb. 2 Einstellungsmittelwerte nach Bildung und Einkommen (Quelle: SOEP-Innovations-Stichprobe 2017; eigene Berechnungen, gewichtet). Bei den Bildungsgruppen wird die Kategorie ,nicht klassifizierbar" nicht dargestellt

Items eingehen. Die so konstruierten Summenindizes werden jeweils durch zwei dividiert, sodass die resultierenden Skalen wieder dem Wertebereich der Items entsprechen. Auch anhand dieser Indexwerte zeigt sich, dass eine deutliche Mehrheit der Befragten Haltungen aufweist, die auf eine Reduktion von Einkommensungleichheit und auf eine Ausweitung der Anerkennung sexueller Diversität gerichtet sind, während sich die Haltungen zur Zuwanderung als deutlich heterogener und polarisierter darstellen (siehe Online-Abbildung 2 im Online-Anhang).

Im nächsten Schritt soll nun analysiert werden, wo sich die unterschiedlichen Statusgruppen auf diesen Einstellungsdimensionen verorten lassen. Hierfür wurden neben einfachen deskriptiven Analysen auch multivariate Regressionsanalysen durchgeführt. Unsere deskriptiven Befunde zeigen, dass in Bezug auf alle drei Einstellungsdimensionen deutliche Unterschiede zwischen den Bildungsgruppen existieren (siehe Abbildung 2 oben): So weisen Hochgebildete im Mittel die stärkste Bereitschaft auf, sexuell diverse Identitäts- und Lebensentwürfe anzuerkennen, und sie sind auch am offensten positioniert, wenn es um das Thema Zuwanderung geht. Hiermit unterscheiden sie sich deutlich von den Geringgebildeten. Bei der ökonomischen Ungleichheit ist das Bild jedoch genau umgekehrt: Hier weisen die Geringgebildeten die kritischsten Einstellungen auf und die Hochgebildeten die unkritischsten. Die Mittelgebildeten sind, wie zu erwarten, in jeder Einstellungsdimension zwischen diesen beiden Gruppen positioniert. Die erwähnten Einstellungsunterschiede bedeuten allerdings nicht, dass Hochgebildete ökonomische Ungleichheiten generell begrüßen oder Geringgebildete generell gegen eine Anerkennung sexueller Diversität sind. Viel eher handelt es sich hier um moderate Gruppenunterschiede, die auf einem 
allgemein hohen Niveau der Ungleichheitskritik bzw. Anerkennungsbereitschaft angesiedelt sind. Lediglich bei den Einstellungen zur Migration - jener Dimension, die allgemein durch eine größere Streuung in der Gesamtbevölkerung gekennzeichnet ist - liegen die Haltungen der Bildungsgruppen so weit auseinander, dass sie im Mittel tatsächlich konträre Positionen einnehmen: Während die Geringgebildeten (und auch die Mittelgebildeten) eher eine Schließung befürworten, weisen die Hochgebildeten hier entgegengesetzte Haltungen auf und schauen deutlich positiver auf Migration bzw. die Inklusion von Migrantinnen und Migranten.

Die Positionierung der Statuslagen in den jeweiligen Einstellungsdimensionen wird noch etwas deutlicher, wenn man die jeweiligen Einstellungsmittelwerte der Bildungsgruppen in einem zweidimensionalen Raum darstellt. Betrachtet man auf diese Weise die Oben-Unten-Ungleichheit und die Wir-Sie-Ungleichheit gleichzeitig, sieht man nach wie vor die oben bereits angesprochenen Unterschiede zwischen den Bildungsgruppen, es wird jedoch ersichtlich, dass alle drei Bildungsgruppen im ungleichheitskritischen bzw. anerkennungsaffinen Quadranten angesiedelt sind (siehe rechter Teil von Abbildung 3a). Erst bei der gemeinsamen Betrachtung von ObenUnten-Ungleichheit und Innen-Außen-Ungleichgeit liegen die Bildungsgruppen in unterschiedlichen Quadranten (siehe rechter Teil von Abbildung 3b): Während die Hochgebildeten im Mittel sowohl kritisch gegenüber ökonomischer Ungleichheit als auch offen bei Migrationsfragen positioniert sind, geht bei den Geringgebildeten (und auch den Mittelgebildeten) eine ungleichheitsaverse Haltung zur ökonomischen Ungleichheit eher mit ablehnenden Haltungen beim Thema Zuwanderung und Inklusion von Nicht-Staatsbürgern in die Sozialsysteme einher. Ein ähnliches Bild ergibt sich schließlich für die gemeinsame Betrachtung von Innen-Außen-Ungleichheit und Wir-Sie-Ungleichheit (siehe rechter Teil von Abbildung 3c).

Für das Einkommen, unseren zweiten Statusindikator, ergeben sich ebenfalls deutliche Unterschiede, die in die gleiche Richtung weisen wie schon die Einstellungsunterschiede der Bildungsgruppen. Hier vertritt die Einkommensoberschicht stärker als die Einkommensunterschicht Haltungen, die auf die Anerkennung sexuell diverser Identitätsformen und auf eine Inklusion von Migranten ausgerichtet sind, während ihre Kritik an der ökonomischen Ressourcenverteilung deutlich schwächer ausgeprägt ist als diejenige der Unterschicht (siehe Abbildung 2 unten). Die Mittelschicht nimmt bei Fragen der Zuwanderung eine Zwischenposition ein, während ihre Haltung bei Fragen der Diversität eher derjenigen der Oberschicht ähnelt und beim Thema der ökonomischen Ungleichheit eher der Haltung der Unterschicht entspricht. Auch hier sind alle betrachteten Einkommensgruppen in Bezug auf die Oben-Unten-Ungleichheit und Wir-Sie-Ungleichheit - im Mittel - im ungleichheitskritischen bzw. anerkennungsbereiten Quadranten positioniert (siehe linker Teil von Abbildung 3a), während sie sich stärker unterscheiden, wenn man die Oben-UntenUngleichheit und Innen-Außen-Ungleichheit in den Blick nimmt: Dann ist es wiederum die Oberschicht, die ungleichheitsavers in beiden Dimensionen auftritt, während die Unterschicht zwar kritisch auf die ökonomische Ungleichheit blickt, aber eher eine ungleichheitsaffine Position bei Migrationsfragen vertritt (siehe linker Teil von Abbildung 3b). Dieses empirische Muster liegt auch bei der gemeinsamen Betrachtung von Innen-Außen-Ungleichheit und Wir-Sie-Ungleichheit vor (siehe linker Teil von Abbildung 3c). 
a

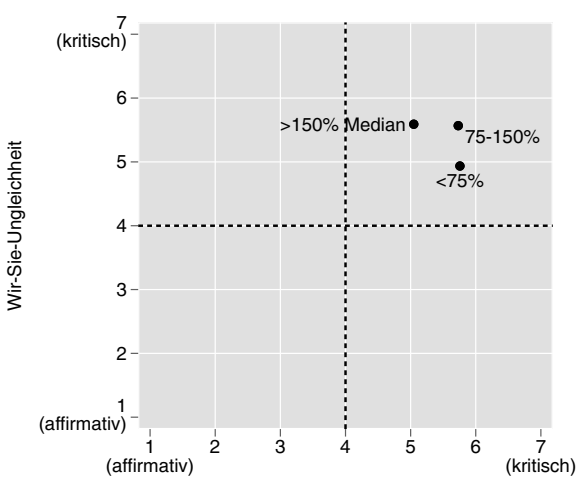

Oben-Unten-Ungleichheit

b

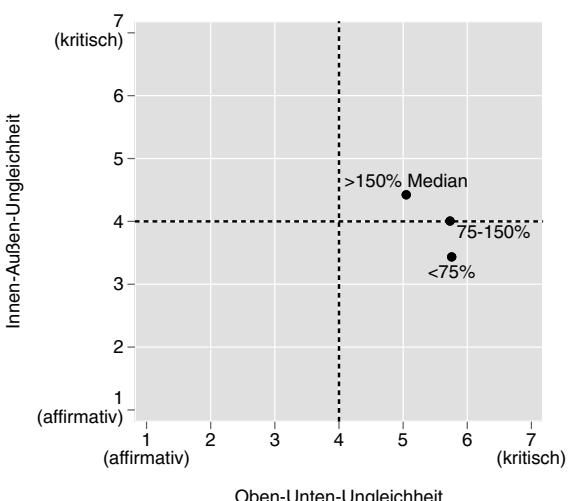

C

Einkommensgruppen

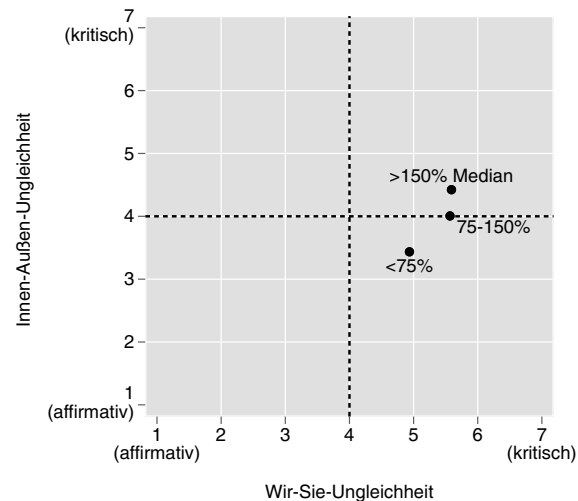

Bildungsgruppen

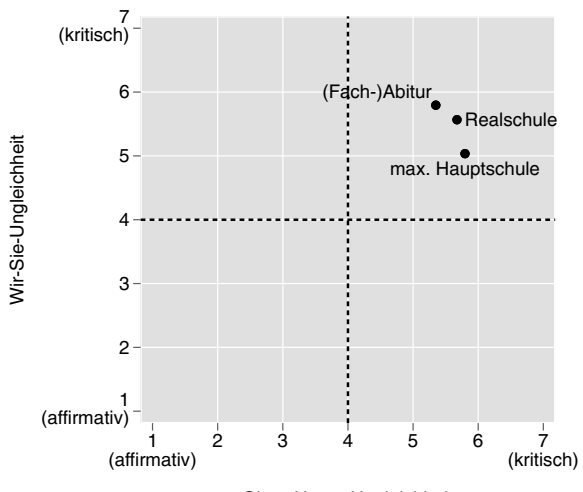

Oben-Unten-Ungleichheit

\section{Bildungsgruppen}

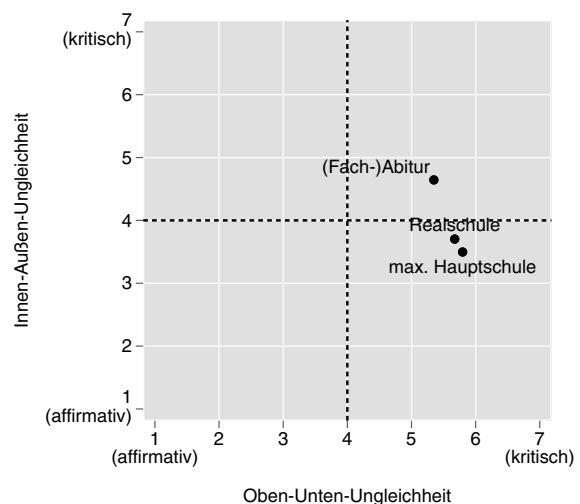

Bildungsgruppen

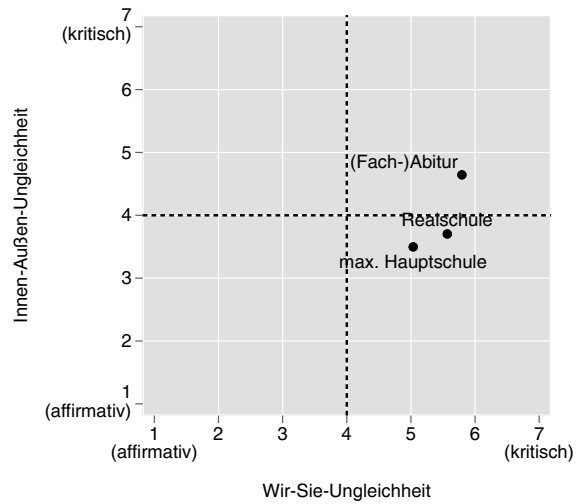

Abb. 3 Einstellungsmittelwerte nach Bildung und Einkommen in zweidimensionaler Betrachtung. (Quelle: SOEP-Innovations-Stichprobe 2017; eigene Berechnungen, gewichtet). Bei den Bildungsgruppen wurde die Kategorie „nicht klassifizierbar“ nicht dargestellt 
Im letzten Schritt wird von uns geprüft, ob die Einstellungsunterschiede zwischen den Bildungs- und Einkommensgruppen signifikant sind und stabil bleiben, wenn man die demografische Komposition der Befragten kontrolliert und beide Statusindikatoren gleichzeitig betrachtet. Hierfür haben wir drei lineare Regressionsmodelle berechnet, in denen jeweils einer der drei Einstellungsindizes die abhängige Variable darstellt. Als zentrale unabhängige Variablen werden Einkommen und Bildung und als Kontrollvariablen die Wohnregion, das Geschlecht, das Alter, der Familienstand und der Migrationsstatus in die Modelle aufgenommen.

Die Ergebnisse dieser Regressionsanalysen (siehe Tabelle 3) zeigen, dass sich die Hochgebildeten und die Einkommensoberschicht in allen drei Einstellungsdimensionen signifikant von den Geringgebildeten und der Einkommensunterschicht unterscheiden: sie haben eine stärkere Präferenz für die Anerkennung sexueller Diversität und für Offenheit gegenüber Migration, ihre Kritik der ökonomischen Ungleichheit ist hingegen schwächer ausgeprägt. Hierbei ist wichtig zu betonen, dass beide Statusindikatoren unabhängig voneinander wirken, d.h. es lässt sich in allen drei Einstellungsdimensionen nicht nur ein Bildungsgradient ausmachen, sondern es ist auch die ökonomische Ressourcenausstattung relevant, die zusätzlich zum Effekt der Bildung wirkt. Beide Effekte lassen sich zudem nicht auf die einbezogenen demografischen Charakteristika der Befragten reduzieren.

Bei den Kontrollvariablen finden wir ein ähnliches Muster in den Einstellungen lediglich für die Wohnregion. Hier zeigen sich Ostdeutsche im Vergleich zu Westdeutschen signifikant kritischer gegenüber ökonomischen Ungleichheiten, aber signifikant zurückhaltender bei der Anerkennung von Diversität und bei der Offenheit gegenüber Migration. Für die übrigen Kontrollvariablen zeigen sich keine signifikanten Effekte über alle drei Einstellungsdimensionen hinweg.

Insgesamt bestätigen sich also unsere Ausgangsannahmen in Bezug auf die Innen-Außen-Ungleichheiten, die wir durch Items zum Zuzug von Ausländern und deren Einbeziehung in die Sozialsysteme erfasst haben. Hier finden wir in der Tat eher demarkationistische Haltungen bei den unteren Statusgruppen und eher apertistische bei den oberen Lagen. Für die Oben-Unten-Ungleichheiten und die WirSie-Ungleichheiten können wir unsere Erwartungen jedoch nicht bestätigen: Zwar nimmt die Zustimmung für eine anerkennungsorientierte Identitätspolitik mit dem sozialen Status zu und die Zustimmung für ökonomische Umverteilung mit dem steigenden sozialen Status ab, jedoch finden wir in unseren empirischen Daten nicht jene klare Polarisierung, von der in den jüngsten Debatten oft die Rede war. Viel eher scheinen die Anerkennung sexueller Diversität und die Einhegung ökonomischer Ungleichheiten Themen zu sein, die statusgruppenübergreifend einen starken Rückhalt in der Bevölkerung haben. Sie besitzen jedenfalls nicht schon von vornherein - wie in der öffentlichen Debatte oft suggeriert - einen geradezu inhärenten Polarisierungscharakter. 
Tab. 3 Einstellungsunterschiede nach sozialstrukturellen Merkmalen (lineare Regressionen, unstandardisierte Regressionskoeffizienten) (Quelle: SOEP-Innovations-Stichprobe 2017; eigene Berechnungen, ungewichtet)

\begin{tabular}{|c|c|c|c|c|c|c|}
\hline \multirow{2}{*}{ Höchster Schulabschlus } & \multicolumn{2}{|c|}{$\begin{array}{l}\text { Innen-Außen-Ungleich- } \\
\text { heit }\end{array}$} & \multicolumn{2}{|c|}{ Wir-Sie-Ungleichheit } & \multicolumn{2}{|c|}{$\begin{array}{l}\text { Oben-Unten-Ungleich- } \\
\text { heit }\end{array}$} \\
\hline & & & & & & \\
\hline - Max. Hauptschule & ref. & & ref. & & ref. & \\
\hline - Realschule & $0,37 * *$ & $(0,001)$ & 0,17 & $(0,157)$ & $-0,16+$ & $(0,063)$ \\
\hline - (Fach-)Abitur & $1,11 * *$ & $(0,000)$ & $0,33 * *$ & $(0,008)$ & $-0,22 *$ & $(0,019)$ \\
\hline $\begin{array}{l}\text { - Nicht klassifizier- } \\
\text { bar }\end{array}$ & 0,16 & $(0,410)$ & $-0,11$ & $(0,624)$ & $-0,07$ & $(0,613)$ \\
\hline \multicolumn{7}{|c|}{ Nettoäquivalenzeinkommen } \\
\hline$-<75 \%$ Median & ref. & & ref. & & ref. & \\
\hline$-75-150 \%$ Median & $0,38 * *$ & $(0,000)$ & $0,36 * *$ & $(0,006)$ & $-0,12$ & $(0,139)$ \\
\hline$->150 \%$ Median & $0,55^{* *}$ & $(0,000)$ & $0,38 *$ & $(0,021)$ & $-0,63 * *$ & $(0,000)$ \\
\hline \multicolumn{7}{|l|}{ Wohnregion } \\
\hline - Westdeutschland & ref. & & ref. & & ref. & \\
\hline - Ostdeutschland & $-0,70 * *$ & $(0,000)$ & $-0,32 *$ & $(0,014)$ & $0,50 * *$ & $(0,000)$ \\
\hline \multicolumn{7}{|l|}{ Geschlecht } \\
\hline - Mann & ref. & & ref. & & ref. & \\
\hline - Frau & 0,01 & $(0,835)$ & $0,56^{* *}$ & $(0,000)$ & $0,18 * *$ & $(0,002)$ \\
\hline \multicolumn{7}{|l|}{ Alter } \\
\hline - 17-29 Jahre & ref. & & ref. & & ref. & \\
\hline - 30-49 Jahre & $-0,27+$ & $(0,057)$ & 0,11 & $(0,444)$ & 0,14 & $(0,220)$ \\
\hline - 50-64 Jahre & $-0,13$ & $(0,428)$ & 0,03 & $(0,868)$ & 0,17 & $(0,171)$ \\
\hline - 65 Jahre oder älter & 0,02 & $(0,915)$ & $-0,40 *$ & $(0,032)$ & 0,15 & $(0,256)$ \\
\hline \multicolumn{7}{|l|}{ Familienstand } \\
\hline - Verheiratet & ref. & & ref. & & ref. & \\
\hline - Ledig & $0,30 *$ & $(0,016)$ & $0,37 * *$ & $(0,006)$ & $-0,06$ & $(0,526)$ \\
\hline - Geschieden & $-0,03$ & $(0,827)$ & $0,24+$ & $(0,081)$ & 0,14 & $(0,167)$ \\
\hline - Verwitwet & $-0,08$ & $(0,612)$ & $-0,52 * *$ & $(0,008)$ & $-0,06$ & $(0,628)$ \\
\hline \multicolumn{7}{|l|}{ Migrationsstatus } \\
\hline $\begin{array}{l}\text { - In D. geb./vor } \\
1949 \text { immigr. }\end{array}$ & ref. & & ref. & & ref. & \\
\hline $\begin{array}{l}\text { - Immigriert nach } \\
1948\end{array}$ & 0,03 & $(0,886)$ & $-1,03 * *$ & $(0,000)$ & $-0,21$ & $(0,165)$ \\
\hline Konstante & $3,33 * *$ & $(0,000)$ & $4,92 * *$ & $(0,000)$ & $5,64 * *$ & $(0,000)$ \\
\hline Fallzahl & 1641 & & 1641 & & 1641 & \\
\hline Korrigiertes $R^{2}$ & 0,14 & & 0,12 & & 0,07 & \\
\hline
\end{tabular}

$p$-Werte in Klammern. $+p<0,10 ; * p<0,05 ; * * p<0,01$

Auf Haushaltsebene geclusterte robuste Standardfehler. Alle abhängigen Variablen haben einen Variationsbereich von 1 (ungleichheitsaffin) bis 7 (ungleichheitsavers). 


\section{Zusammenfassung und Diskussion}

Unser Beitrag hat sich zum Ziel gesetzt, die in der publizistischen und wissenschaftlichen Diskussion oft vertretende These einer neuen gesellschaftlichen Spaltungslinie empirisch unter die Lupe zu nehmen. Dabei geht es um den Gegensatz zwischen den sozialstrukturell höheren Soziallagen und den einfachen, unteren Schichten. Die oberen Statuslagen zählen in dieser Perspektive als Träger des postmaterialistischen Wertewandels und als Globalisierungsgewinner, die auf Grundlage ihrer kulturellen Orientierung und ihrer Ausstattung mit Humankapital für Offenheit, die Anerkennung von Minderheitenrechten und sozialer Diversität sowie für die Pluralisierung von Identitäten eintreten, während sie zugleich eine größere ökonomische Ungleichheitstoleranz aufweisen. Die einfachen und unteren Schichten wünschen sich demgegenüber ökonomische Umverteilung; zugleich sind sie aber skeptisch gegenüber Anerkennungsbestrebungen im Hinblick auf Fragen sexueller Identität und die Inklusion von Gruppen von Ausländerinnen und Ausländern. Die Ausprägung dieser Lager wird oft als neuer großer Kulturkonflikt samt einer entsprechenden sozialstrukturellen Basis beschrieben. Zwar werden diese Thesen einer sich in Konfrontation befindenden Zwei-Lager-Welt immer wieder diskutiert, eine auch nur einfache Validierung auf der Grundlage von Einstellungsdaten erfolgt jedoch oftmals nicht. So bleibt der „Kulturkonflikt“ häufig eine stilisierte These mit nur unvollständiger und schwacher Empirie. Dies gilt vor allem dann, wenn man die Annahmen einer damit verbundenen sozialstrukturellen Polarisierung ins Zentrum stellt.

Unsere Untersuchungsanlage erlaubt es zunächst zu zeigen, dass die Vorstellung eines zweidimensionalen Konfliktraums nicht von den Daten bestätigt wird. Die oft als ein Syndrom verstandenen Fragen der Anerkennung sexueller Diversität und der Einbeziehung von Nicht-Staatsbürgern sind, so lässt sich an unseren Faktorenanalysen ablesen, einstellungsmäßig voneinander zu trennen (wenn auch nicht vollständig unabhängig). Anhand unserer Untersuchung können wir auch zeigen, dass die Einstellungsunterschiede vor allem gradueller Natur sind und sich allenfalls bei der Migrationsfrage eine schroffe Entgegensetzung zwischen Befürwortern und Gegnern von Zuwanderung und Inklusion findet, die den erwarteten Mustern nahekommt. Bei den beiden Fragekomplexen der Zustimmung zu ökonomischer Umverteilung und Ungleichheitsbegrenzung sowie der Gleichstellung homosexueller Paare und der Anerkennung von Transgender-Personen finden wir in allen sozialstrukturellen Gruppen eine mehrheitliche Zustimmung. Die Einstellungsdifferenzen bei diesen Themen bewegen sich in einem moderaten Korridor und weisen nicht auf jene tiefe Spaltungslinie hin, die in der Diskussion vielfach unterstellt wird.

Das bedeutet nicht, dass sich keinerlei Einstellungsunterschiede zwischen den Statusgruppen finden. Gezeigt werden konnte die systematische Variation der Einstellungen, bei der ungleichheitskritische Haltungen in den „neueren“ Konfliktfeldern insbesondere von den oberen Statuslagen vertreten werden, während diese Statuslagen im Hinblick auf die ökonomische Ressourcenverteilung mit ihrer Kritik zurückhaltender sind. Für die unteren Statuslagen ist das empirische Muster genau umgekehrt. Aber noch einmal: Wir finden nur wenige empirische Hinweise auf die in den Gegenwartsdiagnosen oft behauptete Spaltung der Gesellschaft, die sozialstrukturell grundiert ist. Unsere Befunde sind als deutlicher Beleg dafür 
zu verstehen, dass sich bestimmte pauschalierende Thesen über neue gesellschaftliche Konfliktfelder, die aus der Beobachtung politischer Akteure und der öffentlichen Kommunikation abgeleitet werden, nicht notwendigerweise in den Einstellungen unterschiedlicher sozialstruktureller Gruppen spiegeln müssen. Für den publizistischen Diskurs bedeuten unsere Ergebnisse, dass es sich lohnen könnte, empirisch genauer nach Sachthemen zu differenzieren und nicht die Narrative politischer Akteure als Empirieersatz ungeprüft zu übernehmen.

Allerdings sollte man unsere Ergebnisse in dieser Hinsicht nicht überinterpretieren, da sich stärkere Einstellungsunterschiede auch in den Bereichen der ObenUnten-Ungleichheiten und der Wir-Sie-Ungleichheiten zeigen könnten, wenn es um die konkretere Ausgestaltung von politischen Vorschlägen oder die Positionierung in aufgeheizten politischen Diskursen geht. Außerhalb der hier betrachteten Einstellungsebene sind Meinungsdifferenzen und diskursive Zuspitzungen selbstverständlich möglich - im Diskurs selbst werden ja oft Gegnerschaften erzeugt und Differenzen überpointiert. Auch können wir nicht ausschließen, dass andere sozialstrukturelle Differenzierungslinien als die von uns betrachteten eine Rolle spielen. Zudem könnte der Einbezug umfangreicherer Itembatterien weitere (Unter-)Dimensionen der Einstellungskomplexe zutage fördern. Dies würde allerdings unserem Befund, dass der zeitgenössische Konfliktraum mehr als zwei Dimensionen aufweist, nicht widersprechen, sondern ihn eher detaillierter ausbuchstabieren. Insofern sollten zukünftige Studien mit erweiterten Einstellungsskalen für die jeweiligen Ungleichheitsdimensionen arbeiten, deren Einbezug in der vorliegenden Untersuchung leider nicht möglich war. Darüber hinaus wäre in zukünftigen Studien der Einbezug von Items zum Thema Ökologie und Nachhaltigkeit sinnvoll, da sich hier in der allerjüngsten Zeit eine weitere Konfliktlinie abzuzeichnen scheint, bei der es darum geht, inwieweit der gegenwärtige Ressourcenverbrauch die Lebenschancen zukünftiger Generationen beeinträchtigt.

Funding Open Access funding enabled and organized by Projekt DEAL.

Open Access Dieser Artikel wird unter der Creative Commons Namensnennung 4.0 International Lizenz veröffentlicht, welche die Nutzung, Vervielfältigung, Bearbeitung, Verbreitung und Wiedergabe in jeglichem Medium und Format erlaubt, sofern Sie den/die ursprünglichen Autor(en) und die Quelle ordnungsgemäß nennen, einen Link zur Creative Commons Lizenz beifügen und angeben, ob Änderungen vorgenommen wurden.

Die in diesem Artikel enthaltenen Bilder und sonstiges Drittmaterial unterliegen ebenfalls der genannten Creative Commons Lizenz, sofern sich aus der Abbildungslegende nichts anderes ergibt. Sofern das betreffende Material nicht unter der genannten Creative Commons Lizenz steht und die betreffende Handlung nicht nach gesetzlichen Vorschriften erlaubt ist, ist für die oben aufgeführten Weiterverwendungen des Materials die Einwilligung des jeweiligen Rechteinhabers einzuholen.

Weitere Details zur Lizenz entnehmen Sie bitte der Lizenzinformation auf http://creativecommons.org/ licenses/by/4.0/deed.de. 


\section{Anhang}

Tab. 4 Operationalisierung und Verteilung der Variablen (Quelle: SOEP-Innovations-Stichprobe 2017; eigene Berechnungen, ungewichtet)

\begin{tabular}{|c|c|c|c|}
\hline Originalvariable & $\begin{array}{l}\text { Rekodierte } \\
\text { Variable }\end{array}$ & $\begin{array}{l}\text { Relative } \\
\text { Häufig- } \\
\text { keit in } \\
\%\end{array}$ & $\begin{array}{l}\text { Arithm. } \\
\text { Mittel } \\
\text { (Standard- } \\
\text { abw.) }\end{array}$ \\
\hline \multirow[t]{4}{*}{ Alter (metrisch) } & 1: 17-29 Jahre & 13,8 & \\
\hline & 2: 30-49 Jahre & 27,3 & \\
\hline & 3: 50-64 Jahre & 28,0 & \\
\hline & $\begin{array}{l}\text { 4: } 65 \text { Jahre oder } \\
\text { älter }\end{array}$ & 30,9 & \\
\hline \multicolumn{4}{|l|}{ Geschlecht } \\
\hline - Mann & 1: Mann & 47,5 & \\
\hline - Frau & 2: Frau & 52,5 & \\
\hline \multicolumn{4}{|l|}{ Wohnregion } \\
\hline - Alte Bundesländer & $\begin{array}{l}\text { 1: Alte Bundes- } \\
\text { länder }\end{array}$ & 80,1 & \\
\hline - Neue Bundesländer & $\begin{array}{l}\text { 2: Neue Bun- } \\
\text { desländer }\end{array}$ & 19,9 & \\
\hline \multicolumn{4}{|l|}{ Familienstand } \\
\hline - Verheiratet, mit Ehepartner zusammenlebend & 1: Verheiratet & 55,6 & \\
\hline - Verheiratet, dauernd getrennt lebend & Fusioniert mit 1 & & \\
\hline - Ledig, war nie verheiratet & 2: Ledig & 25,2 & \\
\hline $\begin{array}{l}\text { - Geschieden, gleichgeschlechtliche Lebenspartner- } \\
\text { schaft aufgehoben }\end{array}$ & 3: Geschieden & 11,6 & \\
\hline $\begin{array}{l}\text { - Verwitwet, gleichgeschlechtlicher Lebenspartner } \\
\text { verstorben }\end{array}$ & 4: Verwitwet & 7,6 & \\
\hline $\begin{array}{l}\text { - Eingetragene gleichgeschlechtliche Lebenspartner- } \\
\text { schaft, zusammenlebend }\end{array}$ & Fusioniert mit 1 & & \\
\hline $\begin{array}{l}\text { - Eingetragene gleichgeschlechtliche Lebenspartner- } \\
\text { schaft, getrennt lebend }\end{array}$ & Fusioniert mit 1 & & \\
\hline \multicolumn{4}{|l|}{ Schulbildung } \\
\hline - Ohne Abschluss & 1: Gering & 30,0 & \\
\hline - Hauptschulabschluss & Fusioniert mit 1 & & \\
\hline - Realschulabschluss & 2: Mittel & 32,9 & \\
\hline - Fachhochschulreife & 3: Hoch & 28,8 & \\
\hline - Abitur & Fusioniert mit 3 & & \\
\hline - Anderer Abschluss & $\begin{array}{l}\text { 4: Nicht klassi- } \\
\text { fizierbar }\end{array}$ & 8,3 & \\
\hline - Noch Schüler & Fusioniert mit 4 & & \\
\hline - Fehlender Wert & Fusioniert mit 4 & & \\
\hline
\end{tabular}


Tab. 4 (Fortsetzung)

\begin{tabular}{|c|c|c|c|}
\hline Originalvariable & $\begin{array}{l}\text { Rekodierte } \\
\text { Variable }\end{array}$ & $\begin{array}{l}\text { Relative } \\
\text { Häufig- } \\
\text { keit in } \\
\%\end{array}$ & $\begin{array}{l}\text { Arithm. } \\
\text { Mittel } \\
\text { (Standard- } \\
\text { abw.) }\end{array}$ \\
\hline \multirow[t]{4}{*}{$\begin{array}{l}\text { Haushaltsnettoeinkommen } \\
\text { (metrisch; mit multipel imputierten Werten, bereitge- } \\
\text { stellt durch das SOEP-Team) }\end{array}$} & $\begin{array}{l}\text { Gewichtet nach } \\
\text { Haushaltszu- } \\
\text { sammensetzung } \\
\text { (neue OECD- } \\
\text { Skala) }\end{array}$ & & \\
\hline & $\begin{array}{l}\text { Unterschicht } \\
\text { (<75\% Median) }\end{array}$ & 24,3 & \\
\hline & $\begin{array}{l}\text { Mittelschicht } \\
(75-150 \% \\
\text { Median) }\end{array}$ & 57,1 & \\
\hline & $\begin{array}{l}\text { Oberschicht }(> \\
150 \% \text { Median) }\end{array}$ & 18,6 & \\
\hline \multicolumn{4}{|l|}{ Migrationsstatus } \\
\hline - In Deutschland geboren oder vor 1949 immigriert & $\begin{array}{l}\text { 1: In Dt. gebo- } \\
\text { ren/vor } 1949 \\
\text { immigriert }\end{array}$ & 91,6 & \\
\hline - Nicht in Deutschland geboren & $\begin{array}{l}\text { 2: Immigriert } \\
\text { nach } 1948\end{array}$ & 8,4 & \\
\hline $\begin{array}{l}\text { Innen-Außen-Ungleichheit (metrisch, } 1-7 \text { ) } \\
\text { - Heutzutage gibt es in Deutschland zu viele Auslän- } \\
\text { der. } \\
\text { - Personen ohne deutsche Staatsangehörigkeit, die } \\
\text { legal nach Deutschland gekommen sind, sollten die } \\
\text { gleichen Sozialleistungen erhalten wie deutsche } \\
\text { Staatsangehörige. }\end{array}$ & $\begin{array}{l}\text { Additiver Index, } \\
\text { metrisch }(1-7)\end{array}$ & & $\begin{array}{l}3,94 \\
(1,67)\end{array}$ \\
\hline $\begin{array}{l}\text { Wir-Sie-Ungleichheit (metrisch, 1-7) } \\
\text { - Gleichgeschlechtliche Ehen sollten akzeptiert wer- } \\
\text { den. } \\
\text { - Es wäre gut für die Gesellschaft, wenn Transgender- } \\
\text { Personen (Personen, die ihr biologisches Geschlecht } \\
\text { geändert haben) als normal anerkannt werden. }\end{array}$ & $\begin{array}{l}\text { Additiver Index, } \\
\text { metrisch }(1-7)\end{array}$ & & $\begin{array}{l}5,47 \\
(1,78)\end{array}$ \\
\hline $\begin{array}{l}\text { Oben-Unten-Ungleichheit (metrisch, 1-7) } \\
\text { - Die Einkommensunterschiede in Deutschland sind } \\
\text { zu groß. } \\
\text { - Es ist die Aufgabe des Staates, die Einkommens- } \\
\text { unterschiede zwischen den Leuten mit hohem Ein- } \\
\text { kommen und solchen mit niedrigem Einkommen zu } \\
\text { verringern. }\end{array}$ & $\begin{array}{l}\text { Additiver Index, } \\
\text { metrisch }(1-7)\end{array}$ & & $\begin{array}{l}5,64 \\
(1,30)\end{array}$ \\
\hline
\end{tabular}




\section{Literatur}

Adamaczyk, A., \& Liao, Y. (2019). Examining public opinion about LGBTQ-related issues in the United States and across multiple nations. Annual Review of Sociology, 45, 401-423.

Alderson, A. S., \& Nielsen, F. (2002). Globalization and the great U-turn: Income inequality trends in 16 OECD countries. American Journal of Sociology, 107, 1244-1299.

Andersen, R., \& Fetner, T. (2008). Economic inequality and intolerance: Attitudes toward homosexuality in 35 democracies. American Journal of Political Science, 52, 942-958.

Berg-Weger, M. (2016). Social work and social welfare. An invitation. New York: Routledge.

Biskamp, F. (2020). Die Arbeiter_innen, der Autoritarismus und die AfD. Einige Daten aus Runde 9 des European Social Survey. Blog-Beitrag vom 2. Februar 2020. http://blog.florisbiskamp.com/2020/02/ 02/die-arbeiter_innen-der-autoritarismus-und-die-afd/. Zugegriffen: Sept. 2020.

Davidov, E., Meulemann, B., Schwartz, S. H., \& Schmidt, P. (2014). Individual values, cultural embeddedness, and anti-immigration sentiments: Explaining differences in the effect of values on attitudes toward immigration across Europe. Kölner Zeitschrift für Soziologie und Sozialpsychologie, 66, 263-285.

van Dyk, S. (2019). Identitätspolitik gegen ihre Kritik gelesen. Für einen rebellischen Universalismus. Aus Politik und Zeitgeschichte, 69(9-11), 25-32.

van Dyk, S., \& Graefe, S. (2019). Wer ist Schuld am Rechtspopulismus? Zur Vereinnahmung der Vereinnahmungsthese: eine Kritik. Leviathan, 47, 405-427.

Eribon, D. (2016). Rückkehr nach Reims. Berlin: Suhrkamp.

Fatke, M. (2018). Inequality perceptions, preferences conducive to redistribution, and the conditioning role of social position. Societies, $8,99$.

Fernández, J. J., \& Jaime-Castillo, A. M. (2017). The institutional foundation of social class differences in pro-redistribution attitudes: a cross-national analysis, 1985-2010. Social Forces, 96, 1009-1038.

Foroutan, N. (2019). Die postmigrantische Gesellschaft. Ein Versprechen der pluralen Demokratie. Bielefeld: transcript.

Fraser, N. (2017). Vom Regen des progressiven Neoliberalismus in die Traufe des reaktionären Populismus. In H. Geiselberger (Hrsg.), Die große Regression. Eine internationale Debatte über die geistige Situation der Zeit (S. 77-91). Berlin: Suhrkamp.

Fraser, N., \& Honneth, A. (2003). Umverteilung oder Anerkennung? Eine politisch-philosophische Kontroverse. Frankfurt a.M.: Suhrkamp.

Fratzscher, M. (2016). Verteilungskampf. Warum Deutschland immer ungleicher wird. München: Carl Hanser.

Fukuyama, F. (2018). Identity. The demand for dignity and the politics of resentment. New York: Farrar, Straus and Giroux.

Gerhards, J. (2010). Non-discrimination towards homosexuality: The European Union's policy and citizens' attitudes towards homosexuality in 27 European countries. International Sociology, 25, 5-28.

Gerhards, J., Hans, S., \& Carlson, S. (2016). Klassenlage und transnationales Humankapital. Wie Eltern der mittleren und oberen Klassen ihre Kinder auf die Globalisierung vorbereiten. Wiesbaden: Springer VS.

Goodhart, D. (2004). Too diverse? Prospect, 95, 7.

Grabka, M. M., \& Goebel, J. (2018). Einkommensverteilung in Deutschland: Realeinkommen sind seit 1991 gestiegen, aber mehr Menschen beziehen Niedrigeinkommen. DIW Wochenbericht, 85, 449-459.

Grabka, M. M., \& Westermeier, C. (2014). Anhaltend hohe Vermögensungleichheit in Deutschland. DIW Wochenbericht, 81, 151-164.

Groh-Samberg, O. (2014). No Way Out - Dimensionen und Trends der Verfestigung von Armut in Deutschland. Sozialer Fortschritt. Unabhängige Zeitschrift für Sozialpolitik, 63, 307-315.

Groh-Samberg, O., \& Hertel, F. (2015). Ende der Aufstiegsgesellschaft? Aus Politik und Zeitgeschichte, 65(10), 25-31.

Hark, S., \& Villa, P. (Hrsg.). (2015). Anti-Genderismus. Sexualität und Geschlecht als Schauplätze aktueller politischer Auseinandersetzungen. Bielefeld: transcript.

Heath, A.F., \& Tilley, J. R. (2005). British national identity and attitudes towards immigration. International Journal on Multicultural Societies, 7, 119-132.

Heisterhagen, N. (2018). Die liberale Illusion. Warum wir einen linken Realismus brauchen. Bonn: Dietz.

Jörke, D., \& Heisterhagen, N. (2017). Was die Linken jetzt tun müssen. Frankfurter Allgemeine Zeitung vom 26.01.2017, 9 . 
Kitschelt, H. (1994). The transformation of European social democracy. Cambridge: Cambridge University Press.

Kitschelt, H. (2004). Diversification and reconfiguration of party systems in postindustrial democracies. Bonn: Friedrich-Ebert-Stiftung.

Koppetsch, C. (2019). Die Gesellschaft des Zorns. Rechtspopulismus im globalen Zeitalter. Bielefeld: transcript.

Kraemer, K. (2018). Sehnsucht nach dem nationalen Container. Zur symbolischen Ökonomie des neuen Nationalismus in Europa. Leviathan, 46, 280-302.

Kriesi, H., Grande, E., Lachat, R., Dolezal, M., Bornschier, S., \& Frey, T. (2008). West European politics in the age of globalization. Cambridge: Cambridge University Press.

Kunovich, R. M. (2004). Social structural position and prejudice: An exploration of cross-national differences in regression slopes. Social Science Research, 33, 20-44.

Lengfeld, H., \& Dilger, C. (2018). Kulturelle und ökonomische Bedrohung. Eine Analyse der Ursachen der Parteiidentifikation mit der „Alternative für Deutschland“ mit dem Sozio-oekonomischen Panel 2016. Zeitschrift für Soziologie, 47, 181-199.

Liebig, S., \& Wegener, B. (1995). Primäre und sekundäre Ideologien. Ein Vergleich von Gerechtigkeitsvorstellungen in Deutschland und den USA. In H.-P. Müller \& B. Wegener (Hrsg.), Soziale Ungleichheit und soziale Gerechtigkeit (S. 265-293). Opladen: Leske + Budrich.

Lilla, M. (2017a). The once and future liberal: After identity politics. New York: Harper.

Lilla, M. (2017b). Das Scheitern der Identitätspolitik. Trumps Amerika: Lehren für die Linke. Blätter für deutsche und internationale Politik, 62(1), 48-52.

Lipset, S. M. (1959a). Democracy and working-class authoritarianism. American Sociological Review, 24, $482-501$.

Lipset, S. M. (1959b). Political man. The social bases of politics. New York: Doubleday \& Company.

Lux, T. (2011). Jenseits sozialer Klassen? Eine empirische Untersuchung der Individualisierungsthese am Beispiel von Einstellungen zu sozialer Ungleichheit und Wahlverhalten. Zeitschrift für Soziologie, 40, 436-457.

Mau, S. (2003). The moral economy of welfare states: Britain and Germany compared, London: Routledge.

Mau, S. (2007). Transnationale Vergesellschaftung. Die Entgrenzung sozialer Lebenswelten. Frankfurt a.M.: Campus.

Mau, S. (2016). Ungleichheit ist längst kein Monopol der Linken mehr. Frankfurter Allgemeine Sonntagszeitung vom 25.09.2016, 31.

Mau, S., \& Heuer, J.-O. (2016). Wachsende Ungleichheit als Gefahr für nachhaltiges Wachstum. Wie die Bevölkerung über soziale Unterschiede denkt. Bonn: Friedrich-Ebert-Stiftung.

Merkel, W. (2017a). Die populistische Revolte. Kulturpolitische Mitteilungen, (157), 53-56.

Merkel, W. (2017b). Kosmopolitismus versus Kommunitarismus: Ein neuer Konflikt in der Demokratie. In P. Harfst, I. Kubbe \& T. Poguntke (Hrsg.), Parties, governments and elites. The comparative study of democracy (S. 9-23). Wiesbaden: Springer VS.

Mewes, J., \& Mau, S. (2012). Unraveling working-class welfare chauvinism. In S. Svallfors (Hrsg.), Contested welfare states: Welfare Attitudes in Europe and Beyond (S. 119-157). Stanford: Stanford University Press.

Mewes, J., \& Mau, S. (2013). Globalization, socio-economic status and welfare chauvinism: European perspectives on attitudes toward the exclusion of immigrants. International Journal of Comparative Sociology, 54, 228-245.

Milanović, B. (2016). Global inequality. A new approach for the age of globalization. Cambridge: The Belknap Press of Harvard University Press.

Nagoshi, J. L., Adams, K. A., Terrell, H. K., Hill, E. D., Brzuzy, S., \& Nagoshi, C. T. (2008). Gender differences in correlates of homophobia and transphobia. Sex Roles, 59, 521-531.

Noll, H.-H., \& Weick, S. (2012). Nicht einmal jeder Dritte empfindet soziale Unterschiede in Deutschland als gerecht: Analysen zur Entwicklung von Einstellungen zur sozialen Ungleichheit in Deutschland. Informationsdienst Soziale Indikatoren, (48), 6-11.

Norris, P., \& Inglehart, R. (2019). Cultural backlash: Trump, Brexit, and authoritarian populism. Cambridge: Cambridge University Press.

OECD (2018). A broken social elevator? How to promote social mobility. Paris: OECD.

Oesch, D., \& Rennwald, L. (2018). Electoral competition in Europe's new tripolar political space: Class voting for the left, centre-right and radical right. European Journal of Political Research, 57, 783-807.

Otto, A., \& Gugushvili, D. (2020). Eco-social divides in Europe: Public attitudes towards welfare and climate change policies. Sustainability, 12, 404.

Piketty, T. (2014). Capital in the twenty-first century. Harvard: Harvard University Press. 
Pollack, D. (2014). Wahrnehmung und Akzeptanz religiöser Vielfalt in ausgewählten Ländern Europas: Erste Beobachtungen. In Sektion Religionssoziologie der Deutschen Gesellschaft für Soziologie (Hrsg.), Grenzen der Toleranz (S. 13-34). Wiesbaden: Springer VS.

Raijman, R., Semyonov, M., \& Schmidt, P. (2003). Do foreigners deserve rights? Determinants of public views towards foreigners in Germany and Israel. European Sociological Review, 19, 379-392.

Reckwitz, A. (2017). Die Gesellschaft der Singularitäten. Zum Strukturwandel der Moderne. Berlin: Suhrkamp.

Reckwitz, A. (2019). Das Ende der Illusionen. Politik, Ökonomie und Kultur in der Spätmoderne. Berlin: Suhrkamp.

Richter, D., \& Schupp, J. (2015). The SOEP Innovation Sample (SOEP IS). Schmollers Jahrbuch - Journal of Contextual Economics, 135, 389-400.

Roex, K. L., Huijts, T., \& Sieben, I. (2019). Attitudes towards income inequality: „Winners“ versus „losers“ of the perceived meritocracy. Acta Sociologica, 62, 47-63.

Schimank, U. (2018). Rechtspopulistische Mittelschichten als Gefährder gesellschaftlicher Ordnung. Eine theoretische Skizze. In N. Schöneck-Voß \& S. Ritter (Hrsg.), Die Mitte als Kampfzone. Wertorientierungen und Abgrenzungspraktiken der Mittelschichten (S. 217-240). Bielefeld: transcript.

Semyonov, M., Raijman, R., \& Gorodzeisky, A. (2006). The rise of anti-foreigner sentiment in European societies, 1988-2000. American Sociological Review, 71, 426-449.

Semyonov, M., Raijman, R., Tov, A. Y., \& Schmidt, P. (2004). Population size, perceived threat, and exclusion: A multiple-indicators analysis of attitudes toward foreigners in Germany. Social Science Research, 33, 681-701.

Steele, L. G. (2015). Income inequality, equal opportunity, and attitudes about redistribution. Social Science Quarterly, 96, 444-464.

Stegemann, B. (2017). Der liberale Populismus und seine Feinde. Blätter für deutsche und internationale Politik, 62(4), 81-94.

Svallfors, S. (2006). The moral economy of class: Class and attitudes in comparative perspective. Stanford: Stanford University Press.

Tebbe, E. N., \& Moradi, B. (2012). Anti-transgender prejudice: A structural equation model of associated constructs. Journal of Counseling Psychology, 59, 251-261.

Teney, C. (2012). Space matters. The group threat hypothesis revisited with geographically weighted regression. The case of the NPD 2009 electoral success. Zeitschrift für Soziologie, 41, 207-226.

Teney, C., Lacewell, O. P., \& De Wilde, P. (2014). Winners and losers of globalization in Europe: Attitudes and ideologies. European Political Science Review, 6, 575-595.

Walch, S. E., Ngamake, S. T., Francisco, J., Stitt, R. L., \& Shingler, K. A. (2012). The attitudes toward transgendered individuals scale: Psychometric properties. Archives of Sexual Behavior, 41, 1283-1291.

De Wilde, P., Koopmans, R., Merkel, W., Strijbis, O., \& Zürn, M. (Hrsg.). (2019). The struggle over borders: Cosmopolitanism and communitarianism. Cambridge: Cambridge University Press.

Zürn, M. (2020). Zurück zur Sozialistischen Internationale? Replik auf Carsten Nickel, Floris Biskamp und Michael Hartmann. Leviathan, 48, 161-175.

Zweck, B., \& Glemser A. (2019). SOEP-IS 2017. Survey report on the 2017 SOEP Innovation Sample. SOEP Survey Papers 619: Series B. Berlin: Deutsches Institut für Wirtschaftsforschung.

Steffen Mau geb. 1968. Dr. rer. pol., Professor für Makrosoziologie an der Humboldt-Universität zu Berlin. Forschungsschwerpunkte: Soziale Ungleichheit, Grenzforschung, vergleichende Wohlfahrtsstaatsforschung, Sozialstrukturanalyse. Ausgewählte Veröffentlichungen: Lütten Klein. Leben in der ostdeutschen Transformationsgesellschaft, 2019; The metric society. On the quantification of the social world, 2019; Inequality, marketization and the majority class. Why did the European middle classes accept neoliberalism?, 2015. 
Thomas Lux geb. 1979. Ph.D., Mitarbeiter am Lehrbereich Makrosoziologie der Humboldt-Universität zu Berlin. Forschungsschwerpunkte: Sozialstrukturanalyse, Lebensverlaufssoziologie, Alter(n) und Generationen, Einstellungsforschung. Ausgewählte Veröffentlichungen: Die AfD und die unteren Statuslagen. Eine Forschungsnotiz zu Holger Lengfelds Studie Die „Alternative für Deutschland“: eine Partei für Modernisierungsverlierer?, in: Kölner Zeitschrift für Soziologie und Sozialpsychologie, 2018; (mit S. Scherger) By the sweat of their brow? The effects of starting work again after pension age on life satisfaction in Germany and the UK, in: Ageing \& Society, 2017; Jenseits sozialer Klassen? Eine empirische Untersuchung der Individualisierungsthese am Beispiel von Einstellungen zu sozialer Ungleichheit und Wahlverhalten, in: Zeitschrift für Soziologie, 2011.

Fabian Gülzau geb. 1987. Dr. phil., Mitarbeiter am Lehrbereich Makrosoziologie der Humboldt-Universität zu Berlin und im SFB 1265 „Re-Figuration von Räumen“, Teilprojekt „Die Grenzen der Welt: Prozesse von De- und Rebordering in globaler Perspektive“. Forschungsschwerpunkte: Soziale Ungleichheit, Grenzsoziologie, Migrationsforschung, politische Soziologie, Familiensoziologie. Ausgewählte Veröffentlichungen: A paradigm shift in German family policy: Applying a topic model to map reform and public discourse, 1990-2016, in: European Policy Analysis, 2019; Sandkastengespräche im Netz? Leitbilder ,guter Erziehung“ in einem digitalen Elternforum, in: Journal of Family Research, 2018; (mit S. Mau, L. Laube und N. Zaun) The global mobility divide: How visa policies have evolved over time, in: Journal of Ethnic and Migration Studies, 2015. 